\title{
Strategic Market Making and Risk Sharing
}

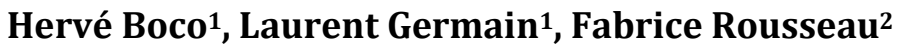 \\ ${ }^{1}$ Toulouse Business School, University of Toulouse, Toulouse, France \\ ${ }^{2}$ Department of Economics, Finance and Accounting, Maynooth University, County Kildare, Ireland \\ Email: h.boco@tbs-education.fr, 1.germain@tbs-education.fr, fabrice.rousseau@nuim.ie
}

How to cite this paper: Boco, H., Germain, L. and Rousseau, F. (2017) Strategic Market Making and Risk Sharing. Journal of Mathematical Finance, 7, 144-179. https://doi.org/10.4236/jmf.2017.71008

Received: October 17, 2016

Accepted: February 3, 2017

Published: February 6, 2017

Copyright (C) 2017 by authors and Scientific Research Publishing Inc. This work is licensed under the Creative Commons Attribution International License (CC BY 4.0).

http://creativecommons.org/licenses/by/4.0/ (c) (†) Open Access

\begin{abstract}
We analyze the result of allowing a risk averse trader to split his order among risk averse market makers. We find that the market makers' aggregate expected utility of profit can increase with the number of market markers and that the aggregate liquidity always increases with it. Despite this latter finding, we show that the cost of trading for the traders increases with the number of market makers as measured by their aggregate expected utility of profit. The larger the market makers' risk aversion, the bigger that cost is. We also find that when the number of market makers tends to infinity, their aggregate expected utility of profit tends to zero. We also obtain that the market makers' individual and aggregate expected utility of profit can increase with their risk aversion and that the trader's expected utility of profit can increase or decrease with the market makers' risk aversion. We offer a potential answer to the ongoing debate concerning the dealers' competitiveness. Indeed, risk aversion reduces competition between market makers as it acts as a commitment for market makers to set higher prices. This commitment is higher the higher the risk aversion.
\end{abstract}

\section{Keywords}

Risk Aversion, Strategic Market Makers, Strategic Traders, Splitting Orders

\section{Introduction}

The competition between markets and the result of that competition has received much attention in Finance. Indeed, traders have the possibility to exchange assets in multiple venues. In addition, companies have a tendency to cross-list more to avail of a greater exposure reducing their cost of capital. In turn, this leads to more markets where traders can trade the same asset. Parallely, after the recent crashes, market participants' attitude toward risk has changed, this has implied an increase in both market participants' risk aversion as well as market volatility. As a consequence, some natural questions arise: How 
is the cost of trading affected by the level of the market makers' risk aversion'? How is that same cost influenced by the number of market makers with whom the traders can exchange? How is the overall liquidity of an asset affected by both the number of market makers and their risk aversion? How is the degree of competition between market makers influenced by the number of traders competing for the exchange of an asset? How is the trading behaviour of risk averse traders affected by the possibility to trade the same asset on different markets or with multiple dealers? These questions need to be answered in order to shed some light on the facts observed in financial markets such as wider bid-ask spreads (high transaction costs), for instance.

In that paper we study the impact of the competition of risk averse market makers on financial markets.

A large body of papers analyzes the formation and properties of price and liquidity in financial markets ${ }^{2}$. In order to study both, three assumptions are commonly made. First, market makers behave competitively. Second, traders cannot split their orders among market makers ${ }^{3}$. Third, market makers are risk neutral. As a result of the first and second assumptions, risk neutral market makers set a price equal to the expected value of the asset given the market maker's information and aggregate order flow. This implies that market makers earn zero expected profit. Both the second and the third assumptions are more simplifying assumptions than realistic ones. Indeed, traders have now a wide range of possibilities to trade a given asset. In addition, [7] proves that, in FX markets, dealers closely control their inventory position showing the fact they are risk averse. It is likely to be also true for equity and bond markets.

In the present model we remove the three aforementioned assumptions. This enables us to combine the assumptions of imperfect competition and risk aversion for the market makers. We then analyze the effects of these assumptions on prices, liquidity and the level of expected profit market makers achieve in a situation where one trader splits his order among market makers.

We propose to answer these questions in a setting close to [2]. The price schedule of a market maker is contingent to the aggregate order flow for that particular market maker only and not contingent to the order flow received by the other market makers. Each market maker determines the price maximizing her expected utility taking as given the price set by her competitors and taking into account its impact on the market order submitted by the trader ${ }^{4}$. Prior to knowing the price schedule, the trader receives (1) a private signal concerning the fundamental value of the asset and (2) an endowment of both the risky asset and the riskless asset. When deciding the size of his order for each market maker, the trader knows the different market makers' price schedule. The trader determines the size of each order submitted to the different markets by maximiz-

${ }^{1}[1]$ raises the fact that too few models study the situation where market makers are risk averse.

${ }^{2}$ Liquidity is defined as the volume necessary to move the price by one unit. See [2].

${ }^{3}$ See [2] [3] [4] [5] [6] among others.

${ }^{4}$ In our context as the price is a linear function of the aggregate order flow it is equivalent to find the level of liquidity maximizing her expected utility. 
ing his conditional expected utility taking into account the impact of his order on the price for each market. We find a counter-intuitive result that increasing the number of market makers, $N$, with whom the trader exchanges, can adversely affect the trader's welfare and this despite the fact that the aggregate liquidity increases with $N$. The interpretation of this result is as follows. Firstly, increasing $N$ has the following effects: (1) it increases the aggregate risk tolerance of the market makers and increases risk sharing, (2) it reduces the individual liquidity in each market, and finally (3) it reduces the volume handled by market makers. The first and the second effect clearly increase aggregate expected utility of profit. However, the reduction in volume has two opposite effects on aggregate expected utility of profit. Secondly, increasing $\rho_{m}$, the market maker's risk aversion, has the following implications: (1) it decreases the aggregate risk tolerance of the market makers, (2) it reduces the individual liquidity in each market, and finally (3) it reduces the volume received by market makers. Effect (1) decreases aggregate expected utility of profit whereas effect (2) increases it. The reduction in volume has again two opposite effects. In fact we show that when $\rho_{m}>0$, the positive effects (those which increase the aggregate expected utility of profit) dominate for a small number of market makers while the negative effect dominates for a large number of market makers. As a result risk aversion can magnify the transaction costs paid by investors. To the best of our knowledge this is the first time this result has been found, as our model looks at the most general situation where both the trader and market makers are strategic and risk averse. This finding has important implications for the regulation of financial markets.

Our result can be regarded as an answer to the ongoing debate about the implications of market fragmentation on traders' welfare. We find that increasing market fragmentation seen as increasing the number of market makers can damage the traders' welfare. Having more market makers or markets is not always desirable from the point of view of investors' trading costs.

Other results include that, for a finite number of market makers, the level of aggregate liquidity is below its competitive level implying that market makers earn positive expected profits ${ }^{5}$. The explanation of that result is as follows: by increasing her price, a market maker reduces the volume received without modifying the proportion of the trader's market order due to hedging needs ${ }^{6}$. However, the increase in price may still compensate for the effect of the decrease in volume on the market maker's expected utility of profit. In fact, despite a higher price, the trader is willing to exchange on that market, as by splitting his order he reduces its overall impact on the price. This implies that, due to their risk aversion, all market makers have an incentive to set less competitive price schedules. Nevertheless, when the number of market makers tends to infinity, both the market makers' expected utility of profit and the aggregate liquidity tend to their competitive level.

${ }^{5}$ The competitive level is computed in a situation where traders cannot split their orders and market makers face competition.

${ }^{6}$ Due the traders' CARA utility framework, an increase in price only alters the size of the market order without changing the proportion of hedging motives within the order. 
Our work is linked to research focusing on dealers' competition. There are strong evidences that dealers behave strategically and earn monopoly rents. [8] and [9] show that market makers on the NASDAQ may exhibit a non-competitive behaviour. This is also confirmed by studies such as [10] and [11]. In an experimental study, [12] find similar results. They compare the size of the bid-ask spread and of the dealers' profit for two scenarios: (1) three competing dealers in a single asset (i.e. direct competition) and (2) three assets with a monopolistic dealer in each (indirect competition). They find that bid-ask spreads are wider and that per-trade dealer profits are larger for the first scenario ${ }^{7}$.

Theoretical papers have looked at the effect of the competition among market makers on their expected profits and their price schedule ${ }^{8}$. [16] and [17] study competition in limit orders. [17] find that when the number of market makers is finite, market makers earn positive expected profits. They also show that as the number of market makers tends to infinity, market makers earn zero expected profits and the price schedule converges to the competitive one obtained in [16]. [18] and [19] consider risk averse market makers. The former compares the cost of trading across markets organized differently, i.e. floors, dealer markets and limit orders. The latter looks at dealership markets, limit order markets and a hybrid market mixing the two preceding structures. They do not provide an analysis of the model we study here. In addition, they look at the case where a unique liquidity trader is present in the market. [20] and [21] look at risk averse market makers, however, their main focus is on an inter-dealer markets. Finally, [22] are closer to our analysis. They study the competition between market makers for the duopoly case. Their setting is similar to [2] with market makers setting price schedules as a function of the aggregate order flow before traders submit their orders. They show that in equilibrium market makers cannot earn zero expected profits. For the duopoly case, the existence and the form of the equilibrium is shown. However, for the oligopolistic case they show that an equilibrium cannot be such that market makers earn zero expected profit but do not prove its existence. We depart from their analysis as we consider the case of risk averse market makers.

The contribution of our paper is twofold. Firstly, on a purely theoretical basis, we generalize [17] and [22] to the cases where there are $N>2$ risk averse market makers. To the best of our knowledge, the dealers' risk aversion has not been incorporated in any analysis for the type of model we are dealing with, i.e. models with asymmetry of information and splitting orders. [4] and [23] analyze the case where market makers are potentially risk averse but traders cannot split their order. Secondly, we offer a potential answer to the ongoing debate concerning the dealers' competitiveness. Indeed, risk aversion reduces competition between market makers as it acts as a commitment for market makers to set

${ }^{7}$ An important difference between the two scenarios lies in the fact that in the three asset case, liquidity traders as well as having the possibility to time their trade have the choice of which asset to trade. This is the main driving force for their result.

${ }^{8}$ Less recent papers [13] [14] [15] focus on the extreme case where the market maker or specialist has a monopolistic position over a particular asset. 
higher prices. This commitment is higher the higher the risk aversion.

An outline of the paper is as follows. In Section 2, we present the general model allowing traders to split their orders. In Section 3, we solve the model for the linear symmetric equilibrium. We look at the properties of the liquidity and the market makers' aggregate expected profit in section 4 . Section 5 presents our conclusions and summarizes our results. Finally all proofs are gathered in the Appendix.

\section{The Model}

Consider a market where a risky asset and a riskless asset are traded among one trader and $N$ market makers. For convenience, the riskless asset has its interest rate normalized to zero. The liquidation value of the risky asset, $\tilde{v}$, is normally distributed with mean 0 and variance $\sigma_{v}^{2}$ (precision $\tau_{v}=\frac{1}{\sigma_{v}^{2}}$ ).

All agents, i.e. the trader and market makers, are risk averse and have preferences described by a CARA utility function of the following form

$$
\begin{gathered}
U(W)=-\exp (-\rho W), \text { for the trader, } \\
U\left(W_{n}\right)=-\exp \left(-\rho_{m} W_{n}\right) \text {, for each market maker } n=1, \cdots, N,
\end{gathered}
$$

where $\rho$ and $\rho_{m}$ represent the parameter of risk aversion and $W$ and $W_{n}$ represent the final wealth.

Before trading the trader receives both an heterogeneous signal about the future value of the risky asset and an heterogeneous endowment of both the risky and the riskless assets. The trader's signal, $s$, is a realization of a normally distributed random variable $\tilde{s}=\tilde{v}+\tilde{\varepsilon}$ where $\tilde{\varepsilon}$ is normally distributed with mean 0 and variance $\sigma_{\varepsilon}^{2}$ (precision $\tau_{\varepsilon}$ ). The trader's endowment of the risky asset, $w$, is a realization of a normally distributed random variable, $\tilde{w}$ with zero mean and variance $\sigma_{w}^{2}$. If $w$ is positive (negative), the trader holds a long (short) position in the risky asset. The trader's endowment of the riskless asset is denoted by $c$. The trader exchanges for two reasons: hedging motives and informational reasons. Indeed, on the one hand, he trades for pure risk-sharing reasons as he receives an endowment shock of the risky asset. On the other hand, as he receives private information he exploits his informational advantage by trading on that private information, he is then an informed speculator. In the present model, we do not require noise traders as part of the orders submitted to the market makers are due to risk sharing motives.

All random variables $\tilde{v}, \tilde{\varepsilon}, \tilde{w}$ are independent.

The timing unfolds as follows:

1) The trader observes his private signal $s$ as well as his endowments, $w$ and $c$ for the risky and the riskless asset, respectively;

2) Each market maker $n=1, \cdots, N$, simultaneously, posts a price schedule depending, solely, on her own order flow. The price schedule is not contingent on the order flow received by the other market makers as it is not observed;

3) Given the market makers' price schedules, the trader determines how much 
to trade with each market maker;

4) Each market maker observes her own aggregate order flow and then clears it at the price previously posted;

5) The value of the asset is revealed and payoffs are realized.

It is assumed that the trader submits market orders in each market.

\section{Characterization of the Equilibrium}

As in [2], the model is solved for linear symmetric equilibria.

We assume that the market order submitted by the trader to market maker $n$ is linear in both the signal and the endowment of the risky asset, i.e.,

$$
x_{n}=a_{n} s-b_{n} w, \forall n=1, \cdots, N .
$$

The price schedule set by market maker $n$ is linear in the anticipated order flow, $x_{n}$, in her own market,

$$
p_{n}=\lambda_{n} x_{n}, \forall n=1, \cdots, N
$$

Definition (Equilibrium) $\left(\lambda_{1}, \cdots, \lambda_{N}\right) \in \mathfrak{R}^{N}$ and $X^{*} \in L_{2}^{N}$ with $X^{*}=\left(X_{1}^{*}, \cdots, X_{n}^{*}, \cdots, X_{N}^{*}\right)$ is an equilibrium if, given the liquidity set by each market maker, the market orders submitted by the trader, $X^{*}$, to the different market makers are such that

$$
X^{*} \in \arg \max _{x_{n} \in \Re} E[W \mid s, w]-\frac{\rho}{2} \operatorname{var}[W \mid s, w]
$$

with $W=w \tilde{v}+\sum_{n=1}^{N} x_{n} \tilde{v}-\sum_{n=1}^{N} p_{n} x_{n}+c$, and given the market order submitted to market $n$ and the liquidity set by the other market makers, the liquidity set by market maker $n, \lambda_{n}$, is such that

$$
\lambda_{n} \in \arg \max E\left[\left(p_{n}\left(x_{n}^{*}\right)-\tilde{v}\right) x_{n}^{*}\right]-\frac{\rho_{m}}{2} \operatorname{var}\left[\left(p_{n}\left(x_{n}^{*}\right)-\tilde{v}\right) x_{n}^{*}\right] .
$$

The trader determines the size of each market orders, $x_{n}^{*}$, submitted to the different markets by maximizing his conditional expected utility taking into account the impact of his orders on the price for each market. Each market maker determines the level of liquidity maximizing her expected utility taking as given the liquidity set by her competitors and taking into account its impact on the market order submitted by the trader.

Given the linearity assumption of the price schedule, computing the price level maximizing the market maker's expected profit is equivalent to computing the liquidity parameter, $\lambda$, maximizing the expected profit. This is used in order to write the above definition.

The model is solved by backward induction: we first solve the traders' program and then solve the market makers' program.

\section{The Equilibrium}

We look at the trader's program first.

Lemma 1. The trader's program, see (3), admits a unique solution given by 


$$
a_{n}=\frac{\tau_{\varepsilon} \prod_{\substack{i=1 \\ i \neq n}}^{N} \lambda_{i}}{\rho \sum_{\substack{j=1 \\ i \neq 1}}^{N} \lambda_{i=1}+2\left(\tau_{\varepsilon}+\tau_{v}\right) \prod_{i=1}^{N} \lambda_{i}} \text {, with } a_{n}=\frac{\tau_{\varepsilon}}{\rho} b_{n} \text {. }
$$

The quantity submitted to any other market, $j \neq n$, is such that $\lambda_{n} a_{n}=\lambda_{j} a_{j}$. Proof. See Appendix.

Given that solution, the market makers maximize the expected utility of profit as defined above. The next proposition gives us the existence and when established the unicity of the solution to the market makers' program.

Proposition 1 If $\rho^{2} \tau_{w}^{-1}>\tau_{\varepsilon}\left(1+\tau_{v}^{-1} \tau_{\varepsilon}\right)$, a linear symmetric equilibrium exists. The form of the linear equilibrium is given by

- The price set by each market maker $n=1, \cdots, N$ is

$$
p_{n}=\lambda(N) x_{n}, \forall n=1, \cdots, N,
$$

- The trader submits to the different market makers a market order of the following form

$$
x(s, w)=a(N)\left(s-\rho \tau_{\varepsilon}^{-1} w\right),
$$

where $a(N)$ and $\lambda(N)$ are defined in the Appendix. Upon having the possibility to trade in $N$ markets, it is always optimal for the trader to trade in all markets.

The unicity of the equilibrium can be formally established for some parameter configurations. Whenever, it cannot be formally established, we prove it numerically (see Appendix).

Proof. See Appendix.

The model studied here is very general. A drawback of such a general model is that closed form solutions cannot be found. The model is then solved by using numerical procedures.

The sufficient condition for the existence of the equilibrium can be interpreted as follows. It states that the hedging motives must outweigh the informational motives for the existence of a linear equilibrium price schedule. Indeed the hedging motives must be large enough to induce, with a linear price schedule, a non-negative expected profit for the market makers'.

The trader's risk aversion as well as the precision of the private information affect both the size of the market order and its composition. Intuitively, by keeping constant the size of the market order, an increase in the trader's risk aversion has a direct effect of increasing the proportion of the market order due to hedging motives whereas an increase of the precision $\tau_{\varepsilon}$ increases the proportion of the market order due to private information. All other parameters affect the size of the order, without changing its composition.

We look at some of the important properties of both the liquidity and the expected utility of profits of the market makers and the trader.

${ }^{9}$ That condition is similar to the one obtained in [14]. [23] also obtains a sufficient condition for the existence of a linear equilibrium. 


\section{Properties of the Equilibrium}

\subsection{Liquidity}

We look at some of the properties of both the individual liquidity, or market depth, i.e. the liquidity set by each market maker, and aggregate liquidity defined as being the sum of all liquidities. In our case, aggregate liquidity is $\sum_{n=1}^{N} \frac{1}{\lambda_{n}}$.

\section{Result 1 (Liquidity)}

1) The individual liquidity can either be increasing with the number of market makers $(N)$ or be a non-monotonic function (first increasing and then decreasing) of $N$. It decreases with the market maker's risk aversion.

2) The aggregate liquidity increases with $N$ whereas it decreases with the market maker's risk aversion.

Proof. See Appendix.

The result is proved using numerical procedures.

The reaction of the individual liquidity to the number of market makers depends on the trader's risk aversion. It firstly increases with $N$ and then decreases with it when the trader's risk aversion is relatively large whereas it increases with $N$ when the trader's risk aversion is relatively small.

As stated in Proposition 1, the trader splits his order across markets in such a way that the marginal trading cost is equalized across markets. As a consequence, the trader submits a smaller quantity to markets with lower liquidity. By setting a higher price, the market maker does not modify the ratio of hedging to informed trading. Indeed the trader reduces the size of his order without altering its composition ${ }^{10}$. Hence, by increasing her price, a market maker reduces the volume received, however, the increase in price may still compensate for the decrease in volume implying higher expected payoff. This gives the intuition for the slope of the individual price schedule. However, we find that the aggregate price schedule faced by the traders has a smaller slope (as defined by the aggregate liquidity) leading to a more competitive aggregate price schedule. This result is also found in [22].

Figure 1 and Figure 2 show the individual liquidity for different values of the market makers' risk aversion as well as for different number of market makers. For an initial low number of market makers, the decrease in individual liquidity (see Figure 1) or the increase (see Figure 2) is sharper for risk neutral than for risk averse market makers. In addition, as the risk aversion increases, the impact of increasing the number of market makers decreases. The decrease in individual liquidity for a large number of market makers is true for risk neutral as well as for risk averse market makers.

The following simulations (Figure 3 and Figure 4) show the levels of aggregate liquidity for risk neutral as well as risk averse market makers.

\footnotetext{
${ }^{10}$ This property is implied by the CARA setting used here. In a different setting, increasing the price (due to a high level of risk aversion of the market makers, for instance) may induce traders to reduce the hedging to information trading ratio implying a closure of the market. In the present setting, this does not happen.
} 


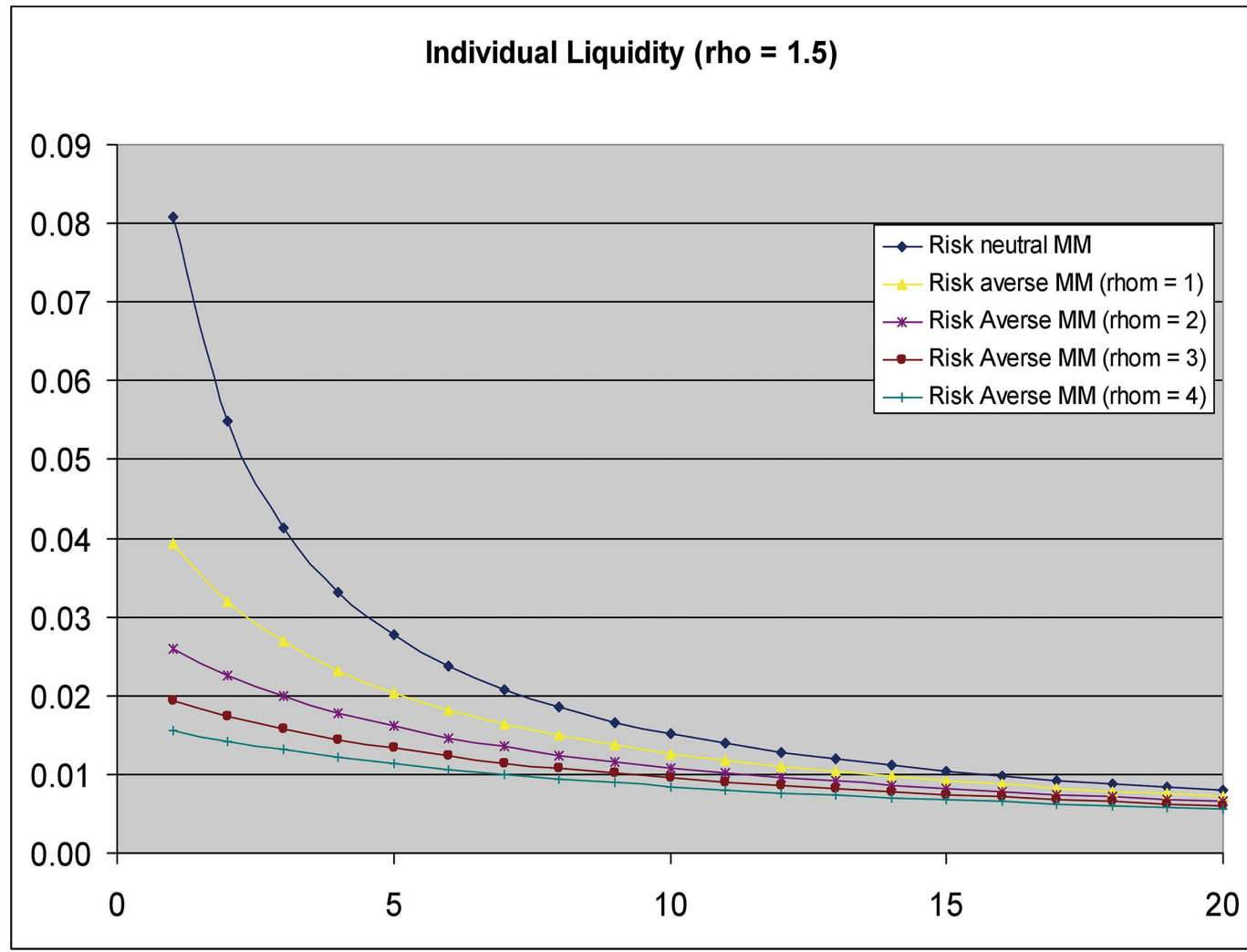

Figure 1. Individual liquidity (liquidity in each market) as a function of the number of market makers $(N)$ and their risk aversion $\left(\rho_{m}\right)$. The figure assumes $\tau_{v}=\tau_{w}=\tau_{\varepsilon}=1$ and $\rho=1.5$.

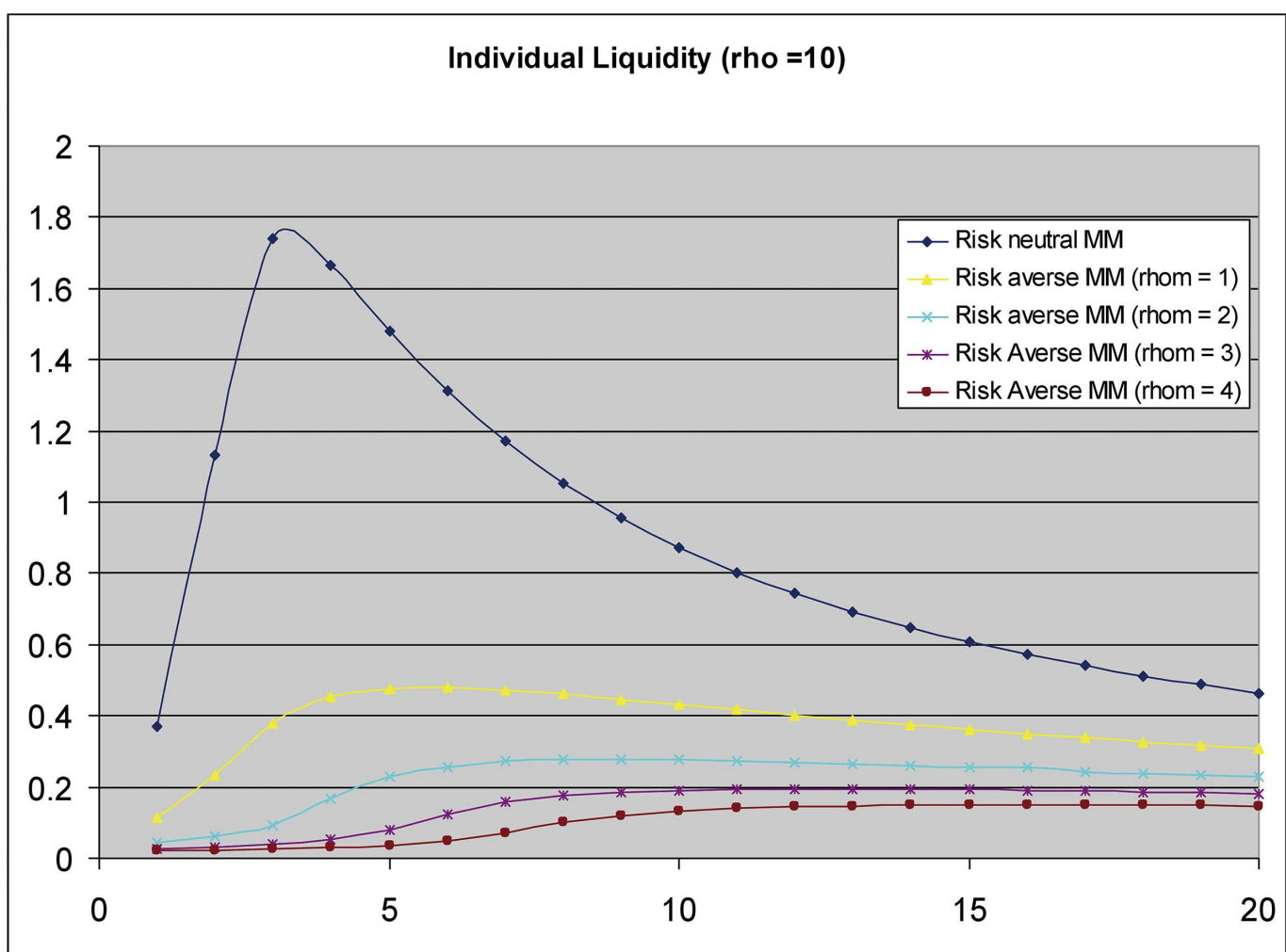

Figure 2. Individual liquidity (liquidity in each market) as a function of the number of market makers $(N)$ and their risk aversion $\left(\rho_{m}\right)$. The figure assumes $\tau_{v}=\tau_{w}=\tau_{\varepsilon}=1$ and $\rho=10$. 


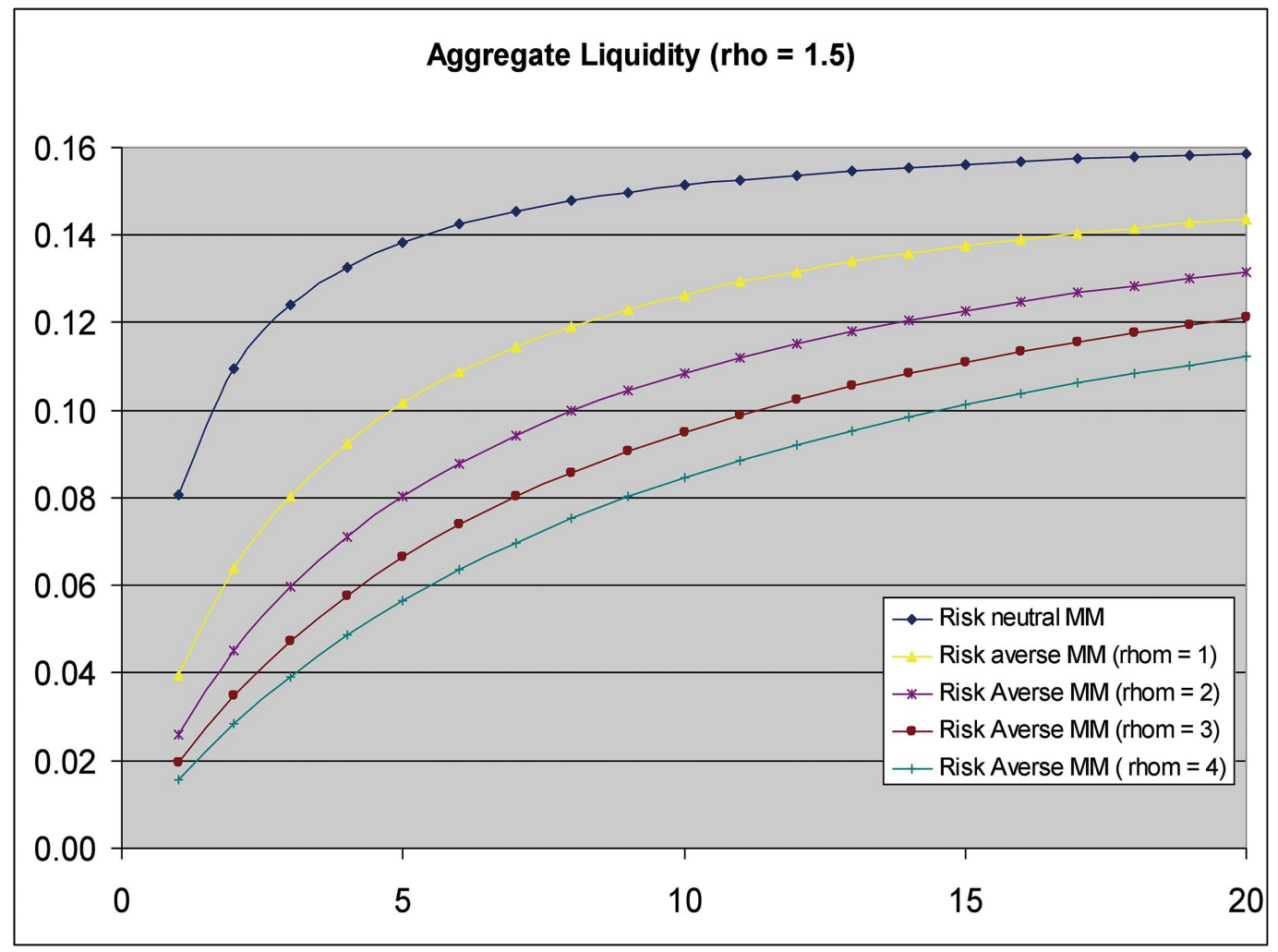

Figure 3. Aggregate liquidity as a function of the number of market makers $(N)$ and their risk aversion $\left(\rho_{m}\right)$. The figure assumes $\tau_{v}=\tau_{w}=\tau_{\varepsilon}=1$ and $\rho=1.5$.

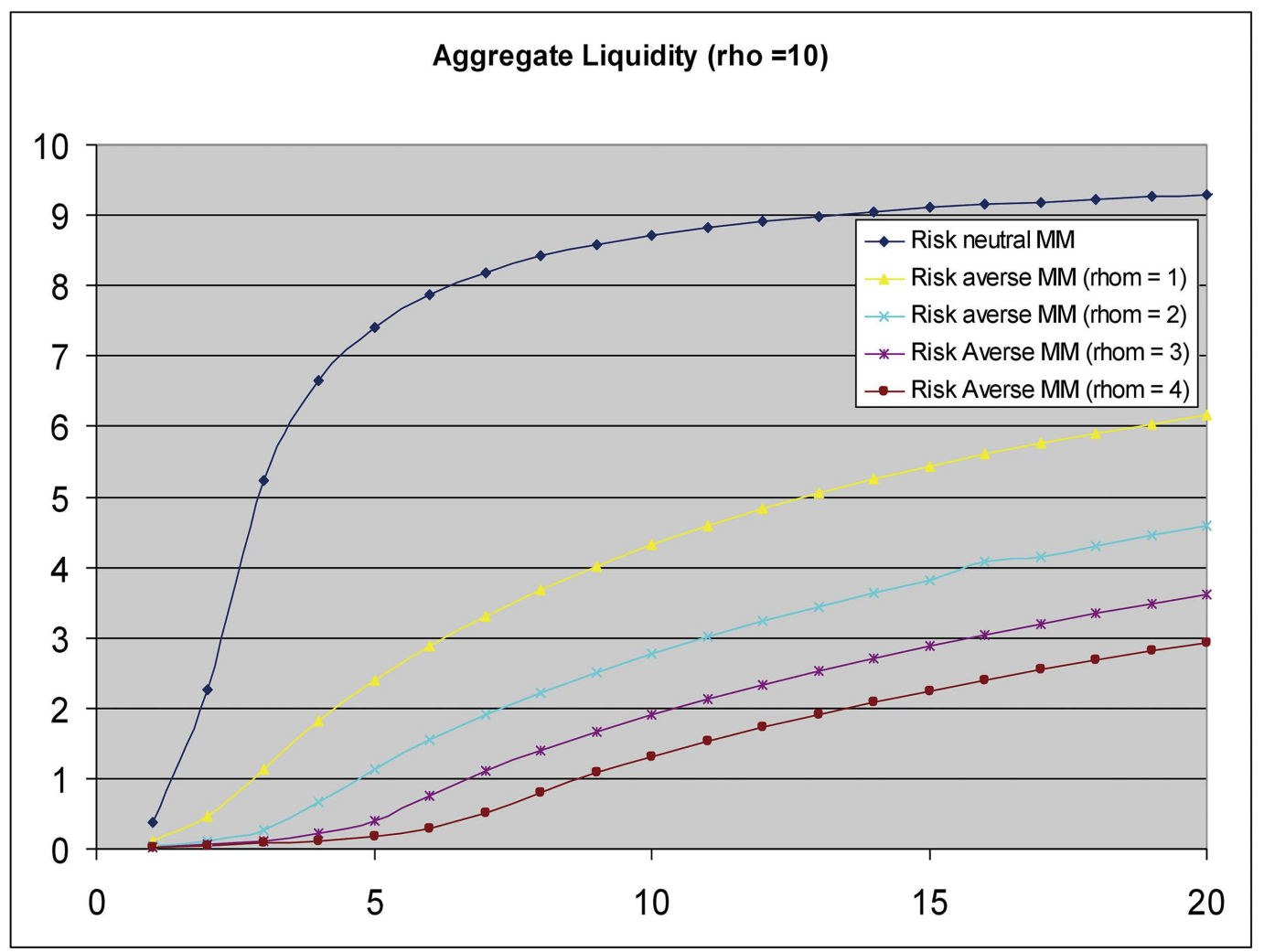

Figure 4. Aggregate liquidity as a function of the number of market makers $(N)$ and their risk aversion $\left(\rho_{m}\right)$. The figure assumes $\tau_{v}=\tau_{w}=\tau_{\varepsilon}=1$ and $\rho=10$. 
From the figures obtained, the aggregate liquidity increases with the number of market makers and converges to the competitive level ${ }^{11}$. The effective price schedule faced by the trader decreases due to more competition. It is also the case, that the aggregate liquidity decreases with the market makers' risk aversion. This comparative static is very intuitive. Indeed, as the market maker's risk aversion increases, the cost of handling a given size of the order flow increases. The market maker then requires more compensation which decreases liquidity and, in turn, reduces the positive impact of competition on the aggregate liquidity level. This can be understood as follows. Risk aversion acts as a commitment device for market makers to set high prices. As their risk aversion increases, their commitment is even stronger reducing the positive impact of competition. The aggregate liquidity increases with the trader's risk aversion. This is explained by the fact that as the trader becomes more risk averse he hedges relatively more than he speculates.

Our model displays some properties consistent with $B M R$ and $B H$ regarding aggregate liquidity and volume traded. They both increase with the number of market makers. It should be pointed out that in $B M R$ the measure of liquidity is the bid-ask spread, they show that it decreases with the number of market makers.

\subsection{Aggregate Expected Utility of Profit}

We now look at the properties concerning the market makers' aggregate expected utility of profit.

\section{Result 2 (Market Maker's Expected Utility of Profit)}

1) In equilibrium, the market makers' individual expected utility of profit decreases with $N$, whereas it is a non-monotonic function of $\rho_{m}$.

2) In equilibrium, the market makers' aggregate expected utility of profit can be a non-monotonic function of both $\rho_{m}$, and $N$.

Proof. See Appendix.

The result is proved using numerical procedures.

Figure 5 and Figure 6 show the individual expected utility of profit for the market makers as a function of $\mathrm{N}$, the number of market makers, and $\rho_{m}$

The market maker's individual expected utility of profit decreases with the number of market makers and tends to zero when the number of market makers is infinite ${ }^{12}$. This clearly results from competition. It can be seen that when the number of market makers is low the individual market makers' expected utility of profit decreases with their level of risk aversion whereas it increases with it when the number of market makers is large enough. This leads to the following result that when the number of market makers is large, it is beneficial for them to be risk averse and the more they are the more beneficial. As explained before, risk aversion acts as a commitment device for market makers to set high prices. As their risk aversion increases, their commitment is even stronger reducing the

${ }^{11}$ This result can be proved analytically for the case of one trader splitting his orders among $N$ risk neutral market makers.

${ }^{12}$ This result is the same as [22]. 


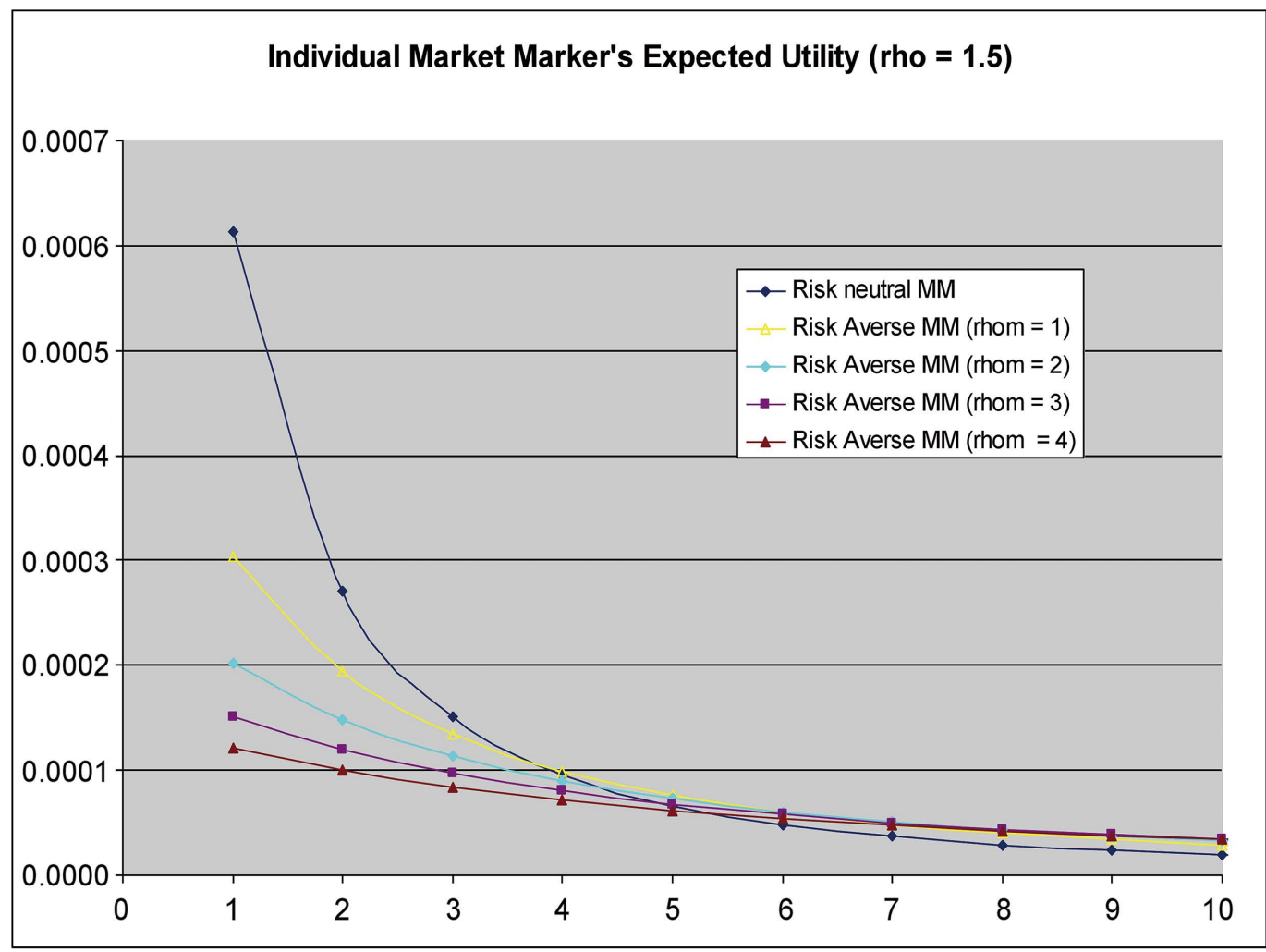

Figure 5. Market maker's expected utility of profit as a function of the number of market makers and their risk aversion. The figure assumes $\tau_{v}=\tau_{w}=\tau_{\varepsilon}=1$ and $\rho=1.5$.

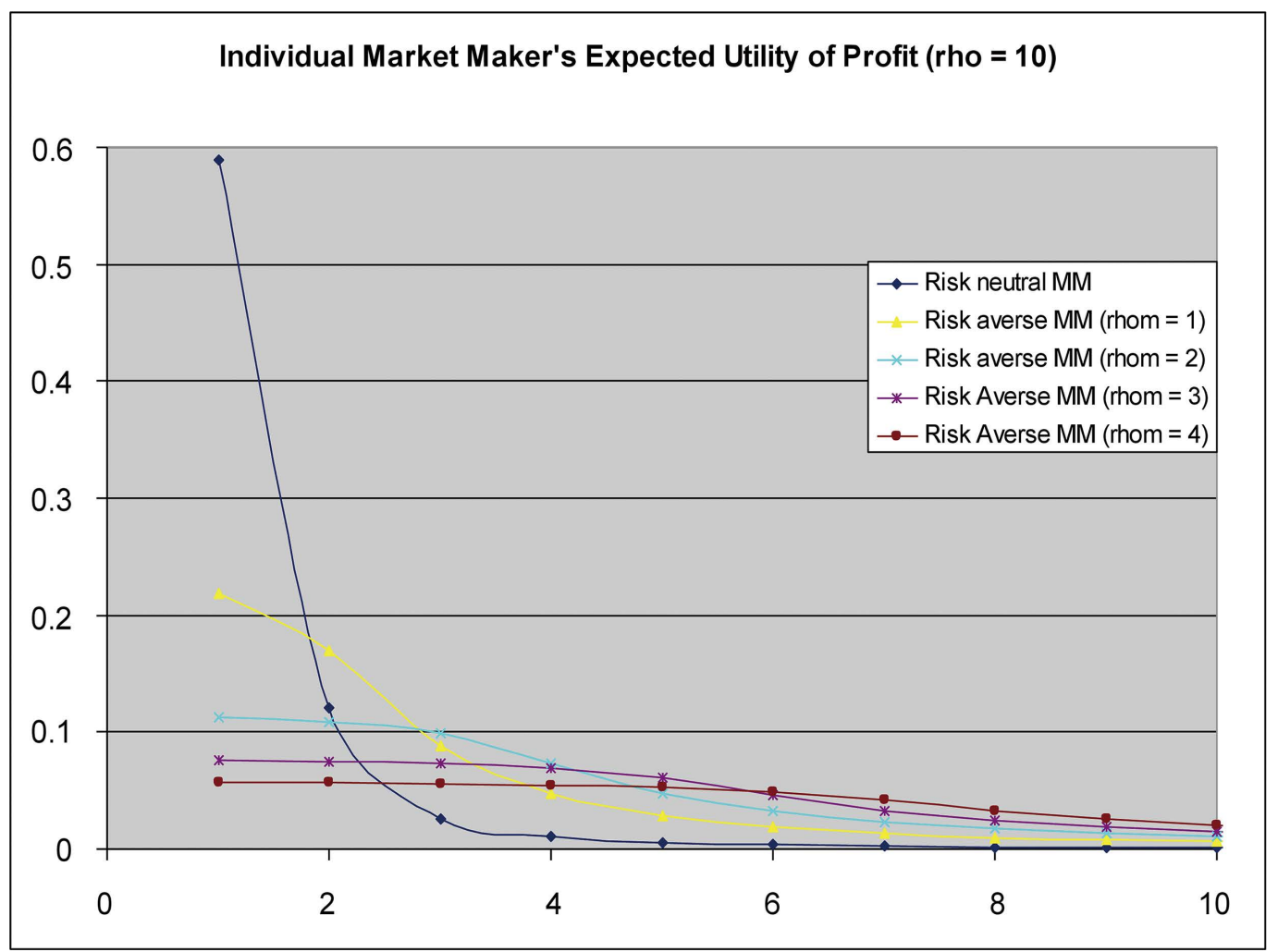

Figure 6. Market maker's expected utility of profit as a function of the number of market makers and their risk aversion. The figure assumes $\tau_{v}=\tau_{w}=\tau_{\varepsilon}=1$ and $\rho=10$. 
positive impact of competition and therefore leading to increasing expected utility with $\rho_{m}$. When the number of market makers is high (very competitive market), risk aversion is beneficial to them as it reduces competition.

Figure 7 and Figure 8 show the aggregate expected utility of profit for the market makers as a function of their number and their risk aversion. The aggregate expected utility of profit for the market makers represents how market makers fare as a group.

As for the individual market maker's expected utility of profit, the aggregate expected utility of profit decreases with $\rho_{m}$ for a low number of market makers and increases with $\rho_{m}$ when the number of market makers is large. The two figures above (7 and 8 ) show a relationship between the optimal number of market makers (number of market makers maximizing the market makers' aggregate expected utility) and their risk aversion. As the level of risk aversion increases, the optimal number of market makers increases.

In order to understand the above results we need to understand all the basic effects on the market makers' aggregate expected utility of profit of changing $N$ and $\rho_{m}$. Firstly, increasing $N$ has the following effects: (1) it increases the aggregate risk tolerance of the market makers and increases risk sharing, (2) it reduces the individual liquidity in each market, and finally (3) it reduces the volume handled by market makers. The first and the second effect clearly increase the market makers' aggregate expected utility of profit. However, the reduction in volume has two opposite effects on aggregate expected utility of profit. Indeed, the reduction in volume has an obvious effect of reducing it but at the

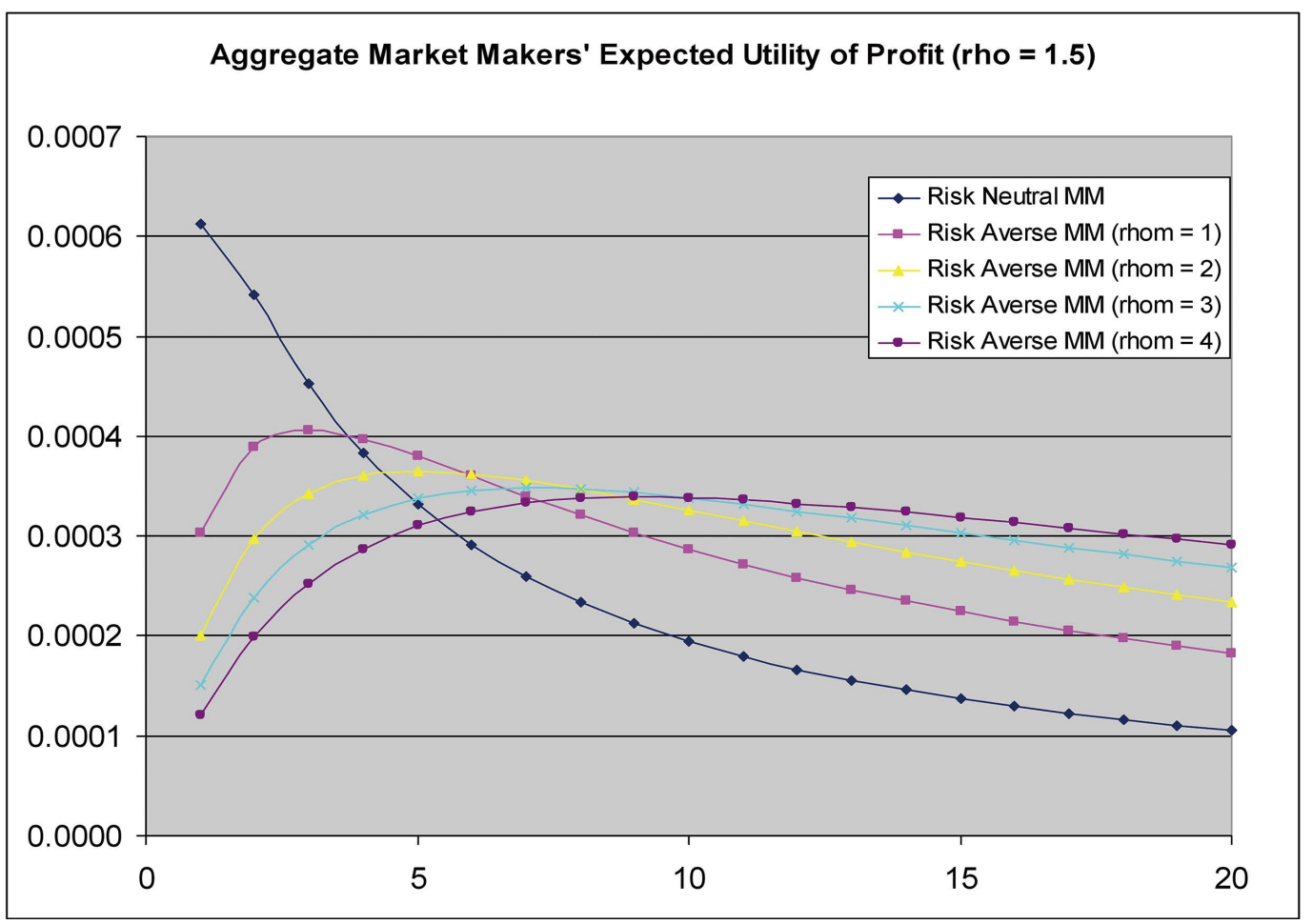

Figure 7. Aggregate market makers' expected utility of profit as a function of the number of market makers and their risk aversion. The figure assumes $\tau_{v}=\tau_{w}=\tau_{\varepsilon}=1$ and $\rho=1.5$. 


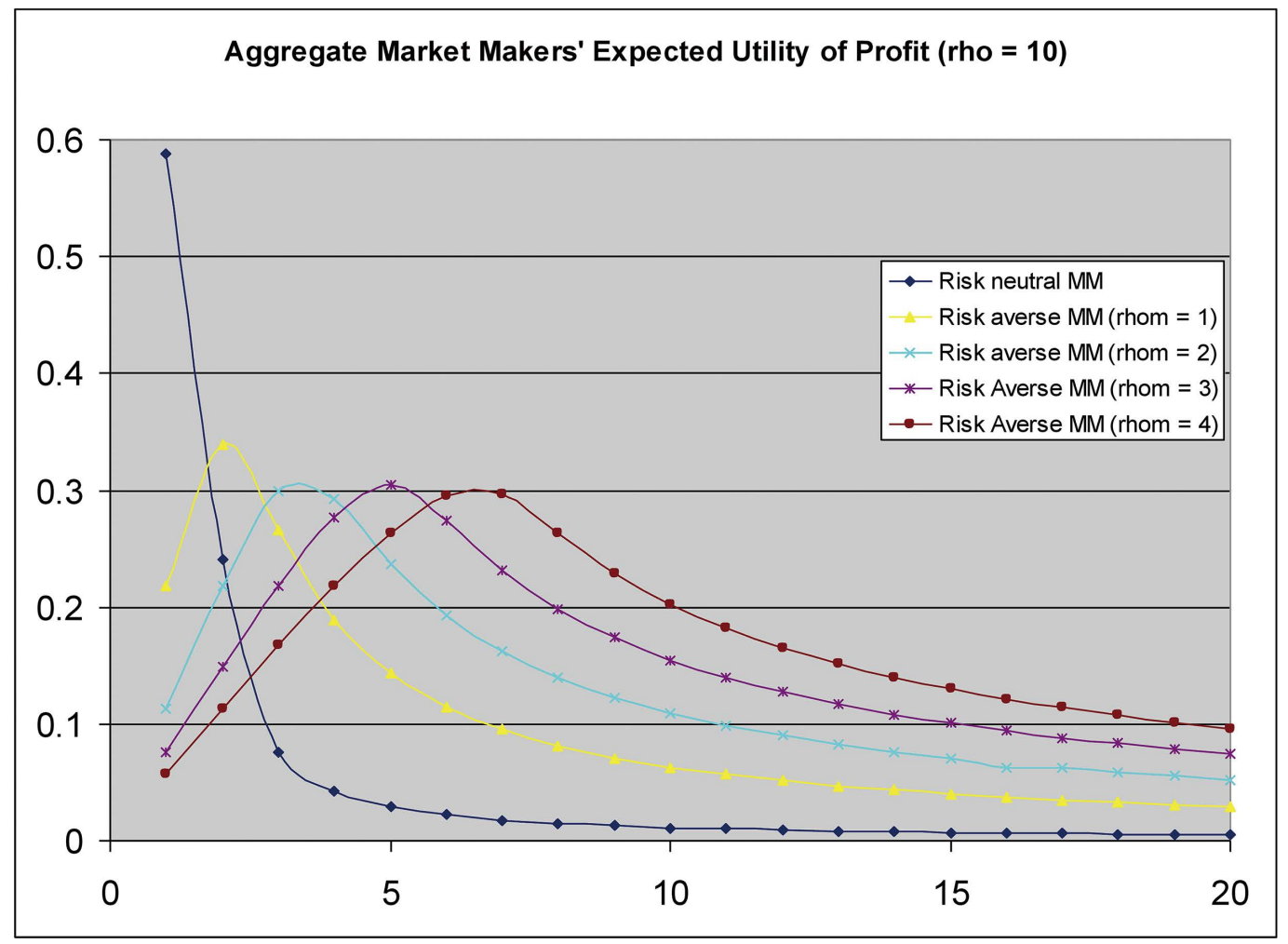

Figure 8. Aggregate market makers' expected utility of profit as a function of the number of market makers and their risk aversion. The figure assumes $\tau_{v}=\tau_{w}=\tau_{\varepsilon}=1$ and $\rho=10$.

same time it reduces the uncertainty faced by market makers increasing it. Secondly, increasing $\rho_{m}$ has the following implications: (1) it decreases the aggregate risk tolerance of the market makers, (2) it reduces the individual liquidity in each market, and finally (3) it reduces the volume received by market makers. Effect (1) decreases aggregate expected utility of profit whereas effect (2) increases it. The reduction in volume has again two opposite effects described earlier.

We now turn to the expected utility of profit for the trader. This provides us with a measure of the overall and true cost of trading for the investor.

Result 3 (Trader's Expected Utility of Profit) In equilibrium, the trader's expected utility of profit is a non-monotonic function of $N, \rho_{m}$ and $\rho$.

Proof. See Appendix.

The result is proved using numerical procedures.

The following Figure 9 and Figure 10 show the trader's expected utility of profit as a function of both the number of market makers and their risk aversion for different level of traders' risk aversion. As there is only one trader, it is also representing the aggregate expected utility.

Increasing the number of market makers adversely affects the cost of trading. Paradoxically, from the point of view of the traders it is not always desirable to increase the number of market makers providing liquidity in the market.

The above result implies that the widely used measure of traders' welfare, i.e. market depth or liquidity, is an inappropriate measure. Indeed, the traders' wel- 
fare can decrease with the number of market makers despite the fact that aggregate liquidity increases.

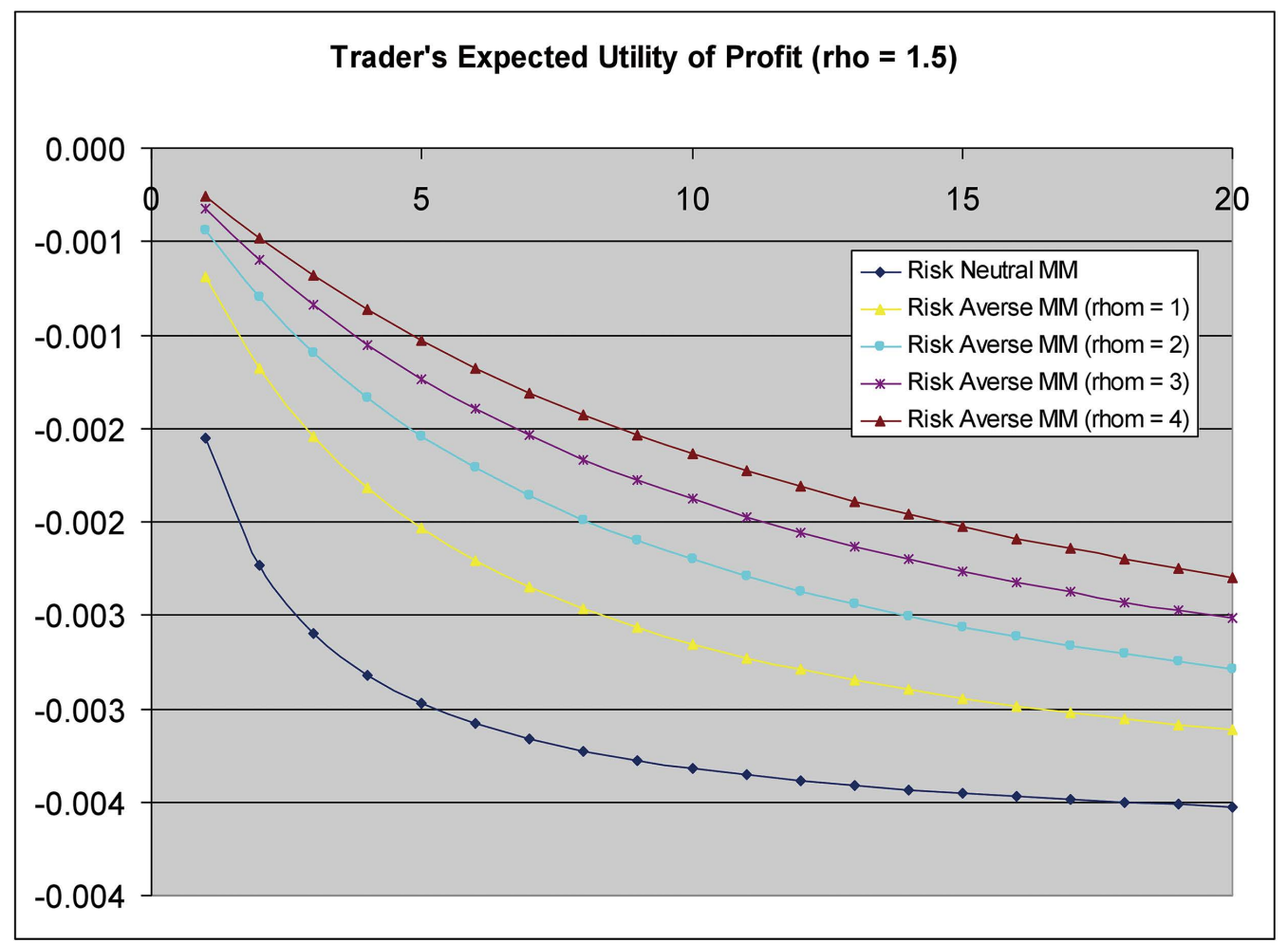

Figure 9. Expected utility of profit of the trader as a function of the number of market makers and their risk aversion. The figure assumes $\tau_{v}=\tau_{w}=\tau_{\varepsilon}=1$ and $\rho=1.5$.

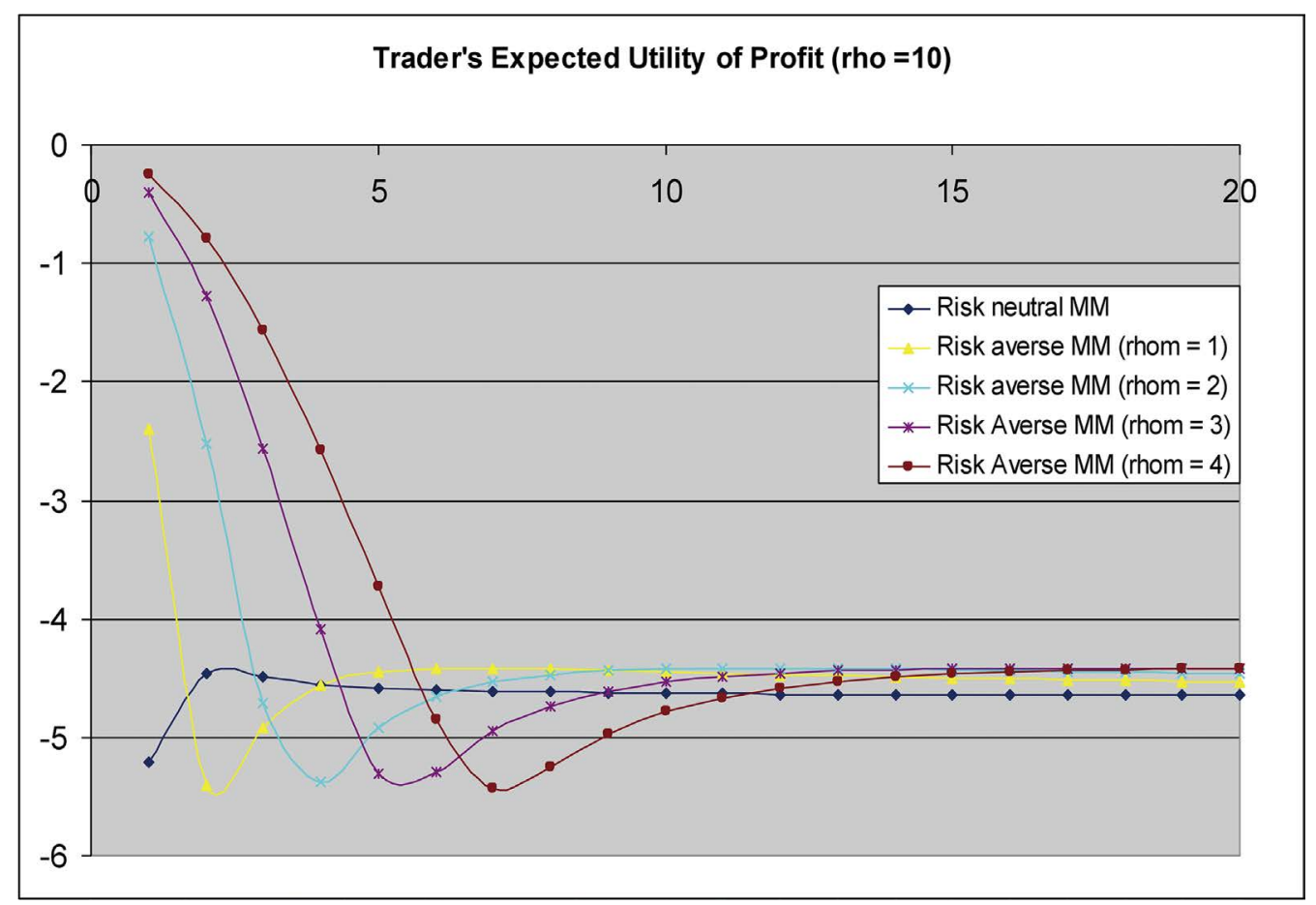

Figure 10. Expected utility of profit of the trader as a function of the number of market makers and their risk aversion. The figure assumes $\tau_{v}=\tau_{w}=\tau_{\varepsilon}=1$ and $\rho=10$. 
In $B M R$, the mark-ups above the competitive or efficient price schedule are shown to decrease with the number of market markers. As market makers are risk neutral this results in a decrease of their expected profit when their number increases. Their result is identical to $B H$.

\subsection{Volatility and Price Efficiency}

We now analyze the impact of having more than one market or market maker onto both the volatility and price efficiency.

\section{Result 4}

1) The price volatility measured as an aggregate of volatility, $\operatorname{var}\left(\sum_{n=1}^{N} p_{n}\right)$ in all markets can either be increasing with $N$ or be a non-monotonic function of $N$. It increases with the market maker's risk aversion and is non-monotonic with the trader's risk aversion.

2) The inverse of the price efficiency is given by

$$
\Sigma_{1}=\frac{\sigma_{v}^{2}\left(\sigma_{\varepsilon}^{2}+\frac{\rho^{2}}{\tau_{\varepsilon}^{2}} \sigma_{w}^{2}\right)}{\sigma_{v}^{2}+\sigma_{\varepsilon}^{2}+\frac{\rho^{2}}{\tau_{\varepsilon}^{2}} \sigma_{w}^{2}} .
$$

It can be shown that it is such that

$$
\frac{1}{2} \sigma_{v}^{2}<\Sigma_{1}<\sigma_{v}^{2} .
$$

It is unaffected by the number of market makers and their risk aversion, whereas it increases with the trader's risk aversion.

Proof. See Appendix.

The next two figures (Figure 11, Figure 12) illustrate the effect of both the number of market makers, $N$, and their risk aversion on price volatility as well as the effect of the trader's risk aversion.

The results on price efficiency follow intuition. As said before, both the number of market makers and their risk aversion have no effect on the composition of the market order (hedging versus speculative motives) leading to the price efficiency being not affected by $N$ and $\rho_{m}$. The trader's risk aversion affects both the size and the composition of the market order. When the trader's risk aversion increases, ceteris paribus the trader increases his hedging motives reducing the price efficiency.

\section{Conclusions}

This paper looks at the case where traders can split their orders among different market makers. Our model combines the assumptions of imperfect competition with risk aversion for the market makers. This study is conducted for a financial market organized as a batch auction. Each market maker commits to a level of liquidity and to a price form, in our case the price is a linear function of the order flow. At that price, each market maker clears the market, i.e., takes a position 


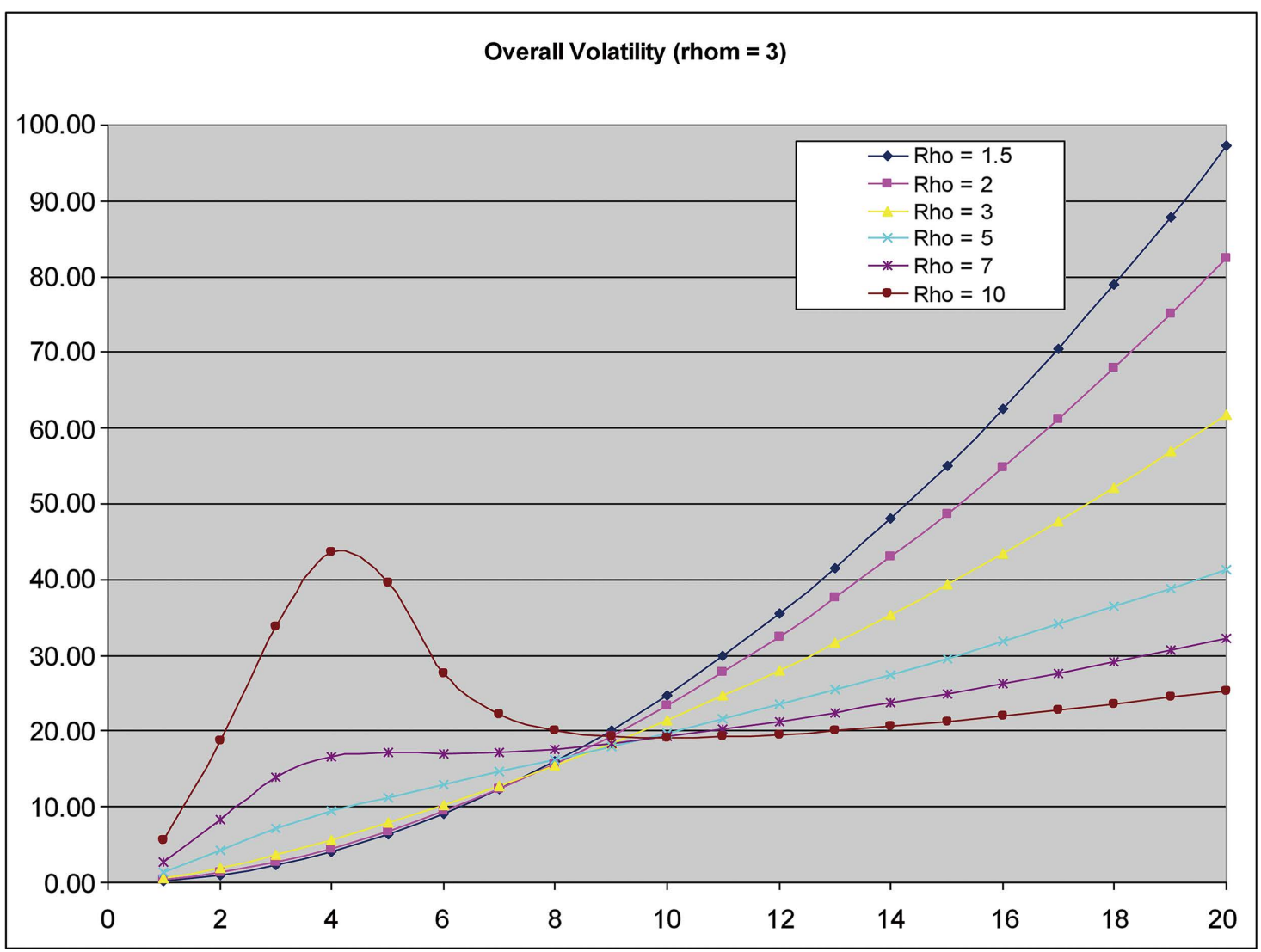

Figure 11. Overall volatility as a function of the number of market makers and their risk aversion. The figure assumes $\tau_{v}=\tau_{w}=\tau_{\varepsilon}=1$ and $\rho=10$.

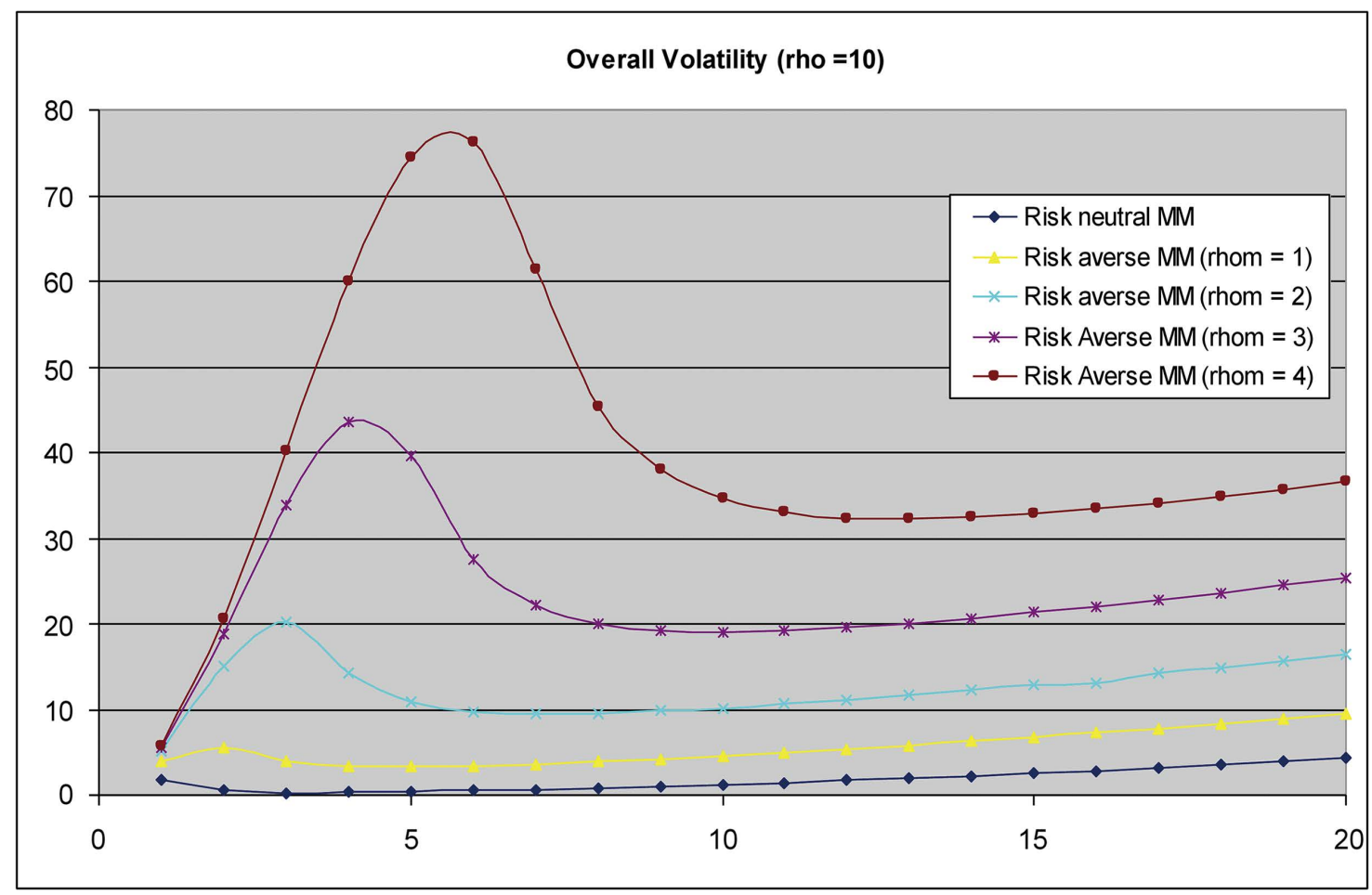

Figure 12. Overall volatility as a function of the number of market makers and their risk aversion. The figure assumes $\tau_{v}=\tau_{w}=\tau_{\varepsilon}=1$ and $\rho=3$. 
that balances supply and demand. The risk averse trader receives both heterogeneous private information of the liquidation value of the traded risky asset and heterogeneous endowment of the same asset. As a consequence, the trader trades for informational as well as hedging motives.

The main findings of the paper are the following. We prove the existence of a linear symmetric equilibrium. We obtain that the aggregate liquidity increases with the number of market makers. For a finite number of market makers, they earn positive expected utility of profit. We show that the market makers' aggregate expected utility of profit can increase with the number of market makers. We also show that the traders' aggregate expected utility of profit can decrease with the number of market makers. This implies that the investors' cost of trading can increase with the number of market makers. As a result the traders' welfare is adversely affected by increasing the number of market makers. A direct implication of that finding is that market liquidity or market depth is an inappropriate measure of investors' trading costs. As in various other papers, it is also shown that market makers' aggregate expected profit tends to zero whenever the number of market makers is infinite.

Empirical studies such as [8] [9] [10] find that market makers on the NASDAQ exhibit a non-competitive behaviour. Our paper brings a new perspective to this non-competitive behaviour. We find that their non-competitive behaviour is exacerbated by their risk aversion. The more risk averse the market makers, the more market makers it takes for the aggregate liquidity to converge to its competitive level. In other words, risk aversion decreases the benefits of competition on the level of aggregate liquidity.

Our results could be regarded as an answer to the ongoing debate about the implications of market fragmentation on traders' welfare. We find that increasing market fragmentation, seen as increasing the number of market makers, can damage traders' welfare. Having more market makers or markets is not desirable from the point of view of investors' trading costs.

\section{Acknowledgements}

We thank the Editor and the anonymous referee for their comments. The second author would like to thank the support he received from the Department DISC at ISAE Supaero where he also lectures. The authors thank the seminar participants at TBS, at Maynooth, and at Amsterdam University. They thank also the useful comments that they got at the AFFI conference and the EFMA conference.

\section{References}

[1] Lyons, R. (2001) The Microstructure Approach to Exchange Rates. MIT Press, Cambridge.

[2] Kyle, A. (1985) Continuous Auctions and Insider Trading. Econometrica, 53, 1315-1335. https://doi.org/10.2307/1913210

[3] Kyle, A. (1984) Market Structure, Information, Futures Markets and Price Formation. In: Storey, G.G., Schmitz, A. and Sarris, A.H., Eds., International Agricultural 
Trade: Advanced Readings in Price Formation, Market Structure, and Price Instabilit, Westview Press, Boulder, 45-64.

[4] Subrahmanyam, A. (1991) Risk Aversion, Market Liquidity, and Price Efficiency. Review of Financial Studies, 4, 417-441. https://doi.org/10.1093/rfs/4.3.417

[5] Foster, F. and Viswanathan, S. (1994) Strategic Trading with Asymmetrically Informed Traders and Long-Lived Information. Journal of Financial and Quantitative Analysis, 29, 499-518. https://doi.org/10.2307/2331107

[6] Vives, X. (1995) The Speed of Information Revelation in a Financial Market Mechanism. Journal of Economic Theory, 67, 178-204.

https://doi.org/10.1006/jeth.1995.1070

[7] Lyons, R. (1995) Test of Microstructural Hypotheses in the Foreign Exchange Market. Journal of Financial Economics, 39, 321-351. https://doi.org/10.1016/0304-405X(95)00832-Y

[8] Christie, W. and Schultz, P. (1994) Why Do Nasdaq Market Makers Avoid OddEight Quotes? Journal of Finance, 49, 1813-1840. https://doi.org/10.1111/j.1540-6261.1994.tb04782.x

[9] Christie, W., Harris, J. and Schultz, P. (1995) Why Did Nasdaq Market Makers Stop Avoiding Odd-Eight Quotes? Journal of Finance, 29, 1841-1860.

[10] Weston, J. (2000) Competition on the Nasdaq and the Impact of Recent Market Reforms. Journal of Finance, 55, 2565-2598.

https://doi.org/10.1111/0022-1082.00300

[11] Simaan, Y., Weaver, D. and Whitcomb, D. (2003) Market Maker Quotation Behavior and Pretrade Transparency. Journal of Finance, 58, 1247-1267. https://doi.org/10.1111/1540-6261.00565

[12] Lamoureux, C. and Schnitzlein, C.R. (2004) Microstructure with Multiple Assets: An Experimental Investigation into Direct and Indirect Dealer Competition. Journal of Financial Markets, 7, 117-143. https://doi.org/10.1016/S1386-4181(03)00030-2

[13] Admati, A. and Pfleiderer, P. (1988) Divide and Conquer: A Theory of Intraday and Day-of-the-Week Mean Effects. Review of Financial Studies, 1, 3-40. https://doi.org/10.1093/rfs/1.1.3

[14] Glosten, L. (1989) Insider Trading, Liquidity, and the Role of the Monopolist Specialist. Journal of Business, 62, 211-235. https://doi.org/10.1086/296460

[15] Dennert, J. (1993) Price Competition Between Market Makers. Review of Economic Studies, 60, 735-751. https://doi.org/10.2307/2298133

[16] Glosten, L. (2003) Is the Electronic Open Limit Order Book Inevitable?. Journal of Finance, 49, 1127-1161.

[17] Biais, B., Martimort, D. and Rochet, J.C. (2000) Competing Mechanisms in a Common Value Environment. Econometrica, 68, 799-837. https://doi.org/10.1111/1468-0262.00138

[18] Biais, B., Foucault, T. and Salanié, F. (1998) Floors, Dealer Markets and Limit Order Markets. Journal of Financial Markets, 1, 253-284. https://doi.org/10.1016/S1386-4181(98)00003-2

[19] Viswanathan, S. and Wang, J. (2002) Market Architecture: Limit-Order Book versus Dealership Markets. Journal of Financial Markets, 5, 127-167. https://doi.org/10.1016/S1386-4181(01)00025-8

[20] Vogler, K. (1997) Risk Allocation and Inter-dealer Trading. European Economic Review, 41, 1615-1634. https://doi.org/10.1016/S0014-2921(96)00049-9 
[21] Lyons, R. (1997) A simultaneous Trade Model of the Foreign Exchange Hot Potato. Journal of International Economics, 42, 275-298. https://doi.org/10.1016/S0022-1996(96)01471-7

[22] Bernhardt, D. and Hughson, E. (1997) Splitting Orders. Review of Financial Studies, 10, 69-101. https://doi.org/10.1093/rfs/10.1.69

[23] Spiegel, M. and Subrahmanyam, A. (1992) Informed Speculation and Hedging in a Noncompetitive Securities Market. Review of Financial Studies, 5, 307-329. https://doi.org/10.1093/rfs/5.2.307 


\section{Appendix}

\section{Proof of Lemma 1}

Proof. Given the different prices set by each market maker $n=1, \cdots, N$, $p_{n}=\lambda_{n} x_{n}$, the investor submits a quantity $x_{n}=a_{n} s-b_{n} w$ to each market. That quantity maximizes the expected profit for the trader taking into account its effect on the price,

$$
\max _{x_{1}, \cdots, x_{N}} E[\tilde{W} \mid \Phi]-\frac{\rho}{2} \operatorname{var}[\tilde{W} \mid \Phi]
$$

with $\tilde{W}=w \tilde{v}+\sum_{n=1}^{N} x_{n} \tilde{v}-\sum_{n=1}^{N} p_{n} x_{n}+c$.

This leads to

$$
\max _{x_{1}, \cdots, x_{N}} w E[\tilde{v} \mid \Phi]+\sum_{n=1}^{N} x_{n} E[\tilde{v} \mid \Phi]-\sum_{n=1}^{N} p_{n} x_{n}+c-\frac{\rho}{2}\left(w+\sum_{n=1}^{N} x_{n}\right)^{2} \operatorname{var}[\tilde{v} \mid \Phi] .
$$

Differentiating the above expression with respect to $x_{n}$, we get $\forall n=1, \cdots, N$

$$
\frac{\partial}{\partial x_{n}}=E[\tilde{v} \mid \Phi]-2 \lambda_{n} x_{n}-\rho\left(w+\sum_{j=1}^{N} x_{j}\right) \operatorname{var}[\tilde{v} \mid \Phi]=0 .
$$

The entire system of first order conditions is given by

$$
D_{N}\left(\begin{array}{c}
x_{1} \\
x_{2} \\
\vdots \\
x_{N}
\end{array}\right)=\left(\begin{array}{c}
1 \\
1 \\
\vdots \\
1
\end{array}\right)(E[v \mid \Phi]-\rho w v a r[v \mid \Phi])
$$

with

$$
\begin{gathered}
D_{N}=\left(\begin{array}{cccc}
C_{1} & D & \cdots & D \\
D & C_{2} & \cdots & D \\
\vdots & \vdots & \ddots & \vdots \\
D & D & \cdots & C_{N}
\end{array}\right), \\
D=\rho \operatorname{var}[v \mid \Phi] \\
C_{i}=2 \lambda_{i}+D .
\end{gathered}
$$

We first prove that the above system admits a unique solution as a maximum using a sequence of steps.

In step 1, we prove a useful property of the above system, i.e. the trader chooses his quantity such that the marginal cost of trading is equal across markets. In step 2, we prove that $D_{N}$ can be inverted, i.e. its determinant is different from zero. In step 3, we prove the existence and unicity of a positive solution. In step 4, we show that the solution is indeed a maximum.

\section{Step 1:}

Lemma 2. $\forall(n, j) \in[1, N] \times[1, N]$ and $n \neq j$, we have that $\lambda_{n} a_{n}=\lambda_{j} a_{j}$ and $\lambda_{n} b_{n}=\lambda_{j} b_{j}$.

Proof. Using the expressions of the market orders as well as 
$E[v \mid \Phi]=\frac{\tau_{\varepsilon} s}{\tau_{\varepsilon}+\tau_{v}}$ and $\operatorname{var}[v \mid \Phi]=\frac{1}{\tau_{\varepsilon}+\tau_{v}}$, the above system (5) can be rewritten as

$$
\left(\begin{array}{ccc}
C_{1} & \cdots & D \\
\vdots & \ddots & \vdots \\
D & \cdots & D \\
D & \cdots & C_{N}
\end{array}\right)\left(\begin{array}{c}
a_{1} s-b_{1} w \\
\vdots \\
\vdots \\
a_{N} s-b_{N} w
\end{array}\right)=\left(\begin{array}{c}
1 \\
\vdots \\
\vdots \\
1
\end{array}\right)\left(\frac{\tau_{\varepsilon} s-\rho w}{\tau_{\varepsilon}+\tau_{v}}\right) .
$$

Looking at the $j$ th line of the above system and identifying the multiplicative parameters for $s$ and $w$ respectively, we get

$$
\begin{aligned}
& \sum_{i=1}^{N} a_{i} D+2 \lambda_{j} a_{j}=\frac{\tau_{\varepsilon}}{\tau_{\varepsilon}+\tau_{v}}, \\
& \sum_{i=1}^{N} b_{i} D+2 \lambda_{j} b_{j}=\frac{\rho}{\tau_{\varepsilon}+\tau_{v}} .
\end{aligned}
$$

Factorizing all terms with $\lambda_{j} a_{j}$ and $\lambda_{j} b_{j}$ for both equations we have

$$
\begin{aligned}
2 \lambda_{j} a_{j} & =\frac{\tau_{\varepsilon}}{\tau_{\varepsilon}+\tau_{v}}-\frac{\rho}{\tau_{\varepsilon}+\tau_{v}} \sum_{i=1}^{N} a_{i}, \\
2 \lambda_{j} b_{j} & =\frac{\rho}{\tau_{\varepsilon}+\tau_{v}}-\frac{\rho}{\tau_{\varepsilon}+\tau_{v}} \sum_{i=1}^{N} b_{i} .
\end{aligned}
$$

From there we can conclude that $\lambda_{j} a_{j}$ for $j=1, \cdots, n$ is equal to a constant and that we have $\lambda_{j} a_{j}=\lambda_{n} a_{n}$ for $n \neq j$, and similarly that $\lambda_{n} b_{n}=\lambda_{j} b_{j}$ for $n \neq j$.

Step 2: We now prove that $D_{N}$ can be inverted.

The matrix $D_{N}$ is given by

$$
D_{N}=\left(\begin{array}{cccc}
2 \lambda_{1}+D & D & \cdots & D \\
D & 2 \lambda_{2}+D & \cdots & D \\
\vdots & \vdots & \ddots & \vdots \\
D & D & \cdots & 2 \lambda_{N}+D
\end{array}\right) .
$$

Lemma 3. $\operatorname{det} D_{N}=2^{N-1} D\left(\sum_{\substack{i=1 \\ N}}^{N} \prod_{\substack{j \neq i \\ j=1}}^{N} \lambda_{j}\right)+2^{N} \prod_{j=1}^{N} \lambda_{j}$.

Proof. The proof is done by iteration.

For $N=1$ and $N=2$, the determinants are given by

$$
\begin{gathered}
\operatorname{det} D_{1}=D+2 \lambda_{1}, \\
\operatorname{det} D_{2}=2 D\left(\lambda_{1}+\lambda_{2}\right)+4 \lambda_{1} \lambda_{2} .
\end{gathered}
$$

It is straightforward to show that both determinants verify the form set in the lemma.

We now show that the form is also true for $N$, assuming that it is true for $N-$ 2 and $N-1$. We rewrite $D_{N}$ as

$$
D_{N}=\left(\begin{array}{cccc}
2 \lambda_{1}+D & D & \ldots & 0 \\
D & 2 \lambda_{2}+D & \ldots & 0 \\
\vdots & \vdots & 2 \lambda_{N-1}+D & -2 \lambda_{N-1} \\
0 & 0 & -2 \lambda_{N-1} & 2 \lambda_{N-1}+\lambda_{N}
\end{array}\right)
$$


where the last column of $D_{N}$ was replaced by the last column minus the $(N-1)$ th column. The same change was performed for the last row.

The determinant by developing from the last line and then from the last column gives

$$
\operatorname{det} D_{N}=2\left(\lambda_{N}+\lambda_{N-1}\right) \operatorname{det} D_{N-1}-4 \lambda_{N-1}^{2} \operatorname{det} D_{N-2} .
$$

Using the form of $\operatorname{det} D_{N-1}$ and $\operatorname{det} D_{N-2}$, and reorganizing the resulting expression we get

$$
\begin{aligned}
\operatorname{det} D_{N}= & 2^{N-1} D\left(\sum_{i=1}^{N-1 N-1} \prod_{\substack{j \neq i \\
j=1}}^{N-1}\left(\lambda_{N-1}+\lambda_{N}\right)-\sum_{i=1}^{N-2 N-2} \prod_{\substack{j \neq i \\
j=1}}^{N} \lambda_{j} \lambda_{N-1}^{2}\right) \\
& +2^{N}\left(\prod_{j=1}^{N-1} \lambda_{j}\left(\lambda_{N-1}+\lambda_{N}\right)-\prod_{j=1}^{N-2} \lambda_{j} \lambda_{N-1}^{2}\right) .
\end{aligned}
$$

After some algebra on both the first and the second term in brackets respectively, we can rewrite them as follows

$$
\begin{gathered}
\sum_{i=1}^{N-1} \prod_{\substack{j \neq i \\
j=1}}^{N-1} \lambda_{j}\left(\lambda_{N-1}+\lambda_{N}\right)-\sum_{i=1}^{N-2} \prod_{\substack{j \neq i \\
j=1}}^{N-2} \lambda_{j} \lambda_{N-1}^{2}=\sum_{i=1}^{N} \prod_{\substack{j i j \\
j=1}}^{N} \lambda_{j}, \\
\prod_{j=1}^{N-1} \lambda_{j}\left(\lambda_{N-1}+\lambda_{N}\right)-\prod_{j=1}^{N-2} \lambda_{j} \lambda_{N-1}^{2}=\prod_{j=1}^{N} \lambda_{j} .
\end{gathered}
$$

Using the latter expressions, the determinant of $D_{N}$ is equal to

$$
\operatorname{det} D_{N}=2^{N-1} D \sum_{i=1}^{N} \prod_{\substack{j \neq i \\ j=1}}^{N} \lambda_{j}+2^{N} \prod_{j=1}^{N} \lambda_{j},
$$

which is the form we were looking for. Moreover the determinant is strictly positive as the $\lambda$ 's are positive. We can then conclude that the matrix can be inverted.

\section{Step 3: Existence and Unicity.}

Given step 1 and step 2, it is straightforward to show that $a_{n}=\frac{\tau_{\varepsilon}}{\rho} b_{n}$ for $n=1, \cdots, N$.

Moreover given step 1, step 2 and the above, the first order condition (4) can be written as

$$
x_{n k}=\frac{A}{2 \lambda_{n}},
$$

with

$$
A=E[\tilde{v} \mid \Phi]-\rho\left(w_{k}+\sum_{j=1}^{N} X_{j k}\left(1-\lambda_{j} a_{j}(K-1)\right)\right) \operatorname{var}\left[\tilde{v} \mid \Phi_{k}\right] .
$$

Given step 1, A independent of $\mathrm{n}$ and is therefore a constant. In the expression defining A, we replace all $x_{i} \forall i=1, \cdots, N$ by $\frac{A}{2 \lambda_{i}}$ and all $b_{n}$ by $a_{n} \frac{\rho}{\tau_{\varepsilon}}$ and put in factor the term $A$ and simplify 


$$
A\left\{1+\rho \operatorname{var}[\tilde{v} \mid \Phi]\left(\sum_{j=1}^{N} \frac{1}{2 \lambda_{j}}\right)\right\}=(E[\tilde{v} \mid \Phi]-\rho \operatorname{var}[\tilde{v} \mid \Phi] w) .
$$

The term multiplied by $A$, henceforth called $H$, can be simplified as follows. We multiply that term by $\prod_{i=1}^{N} \lambda_{i}$. We then have

$$
\prod_{i=1}^{N} \lambda_{i} H=\prod_{i=1}^{N} \lambda_{i}+\rho \operatorname{var}[\tilde{v} \mid \Phi] \prod_{i=1}^{N} \lambda_{i} \sum_{j=1}^{N} \frac{1}{2 \lambda_{j}} .
$$

The last term on the RHS can be simplified as follows using some basic algebra

$$
\begin{aligned}
\rho \operatorname{var}[\tilde{v} \mid \Phi] \prod_{i=1}^{N} \lambda_{i} \sum_{j=1}^{N} \frac{1}{2 \lambda_{j}} & =\frac{\rho \operatorname{var}[\tilde{v} \mid \Phi]}{2} \prod_{i=1}^{N} \lambda_{i} \sum_{j=1}^{N} \frac{1}{\lambda_{j}} \\
& =\frac{\rho \operatorname{var}[\tilde{v} \mid \Phi]}{2} \sum_{j=1}^{N} \frac{\prod_{i=1}^{N} \lambda_{i}}{\lambda_{j}}=\frac{\rho \operatorname{var}[\tilde{v} \mid \Phi]}{2} \sum_{\substack { j=1 \\
\begin{subarray}{c}{i=1 \\
i \neq j{ j = 1 \\
\begin{subarray} { c } { i = 1 \\
i \neq j } }\end{subarray}}^{N} \lambda_{i} .
\end{aligned}
$$

Using the above and the expression of $\operatorname{var}[\tilde{v} \mid \Phi]$, we can rewrite $\prod_{i=1}^{N} \lambda_{i} H$ as

$$
\sum_{j=1}^{N} \prod_{\substack{i=1 \\ i \neq j}}^{N} \lambda_{i}\left(\frac{\rho}{2\left(\tau_{\varepsilon}+\tau_{v}\right)}\right)+\prod_{i=1}^{N} \lambda_{i} .
$$

It is straightforward to see that $H$ is positive.

Using all the simplifications, Equation (8) leads to

$$
2 \lambda_{n} x_{n}=\frac{\left(\tau_{\varepsilon} s-\rho w\right) \prod_{i=1}^{N} \lambda_{i}}{\left(\tau_{\varepsilon}+\tau_{v}\right) \prod_{i=1}^{N} \lambda_{i} H},
$$

Given the expression of $x_{n}$, by identification we obtain the unique solution for $a_{n}$ given by

$$
a_{n}=\frac{\tau_{\varepsilon} \prod_{\substack{i=1 \\ i \neq n}}^{N} \lambda_{i}}{\rho \sum_{\substack{j=1 \\ i \neq 1 \\ i \neq j}}^{N} \lambda_{i}+2\left(\tau_{\varepsilon}+\tau_{v}\right) \prod_{i=1}^{N} \lambda_{i}}>0 .
$$

Step 4: In order to prove that the solution is a maximum we prove that $-D_{N}$ is negative semidefinite. That matrix is given by

$$
D_{N}=\left(\begin{array}{cccc}
-2 \lambda_{1}-D & -D & \cdots & -D \\
-D & -2 \lambda_{2}-D & \cdots & -D \\
\vdots & \vdots & \ddots & \vdots \\
-D & -D & \cdots & -2 \lambda_{N}-D
\end{array}\right)
$$

It can be seen that $\operatorname{det}\left(-D_{N}\right)=(-1)^{N} \operatorname{det} D_{N}$. From the Lemma proved in step 2, we know that det $D_{N}>0$, which implies for uneven $N$ that $\operatorname{det}\left(-D_{N}\right)<0$, where as for even $N \operatorname{det}\left(-D_{N}\right)>0$. This proves that the matrix 
$-D_{N}$ is negative semidefinite which in turn proves that the solution is a maximum.

The two following Lemmas give the expression of the expected utility of profit for market maker $n$ and the expression of the expected utility for trader $n$ respectively. These expressions are used in the numerical procedures.

Lemma 4. Given the linearity of both the market orders and the price schedule, given by (1) and (2) respectively, the expected utility of profit for market maker $n$ is given by

$$
\begin{aligned}
\Pi_{n}= & a_{n} \sigma_{v}^{2}\left(\lambda_{n} a_{n}-1\right)+\lambda_{n} a_{n}^{2}\left(\sigma_{\varepsilon}^{2}+\left(\frac{\rho}{\tau_{\varepsilon}}\right)^{2} \sigma_{w}^{2}\right)-\frac{\rho_{m}}{2} a_{n}^{2}\left[2 \sigma_{v}^{4}\left(\lambda_{n} a_{n}-1\right)^{2}\right. \\
& \left.+\left(\sigma_{\varepsilon}^{2}+\left(\frac{\rho}{\tau_{\varepsilon}}\right)^{2} \sigma_{w}^{2}\right)\left(\sigma_{v}^{2}\left(2 \lambda_{n} a_{n}-1\right)^{2}+2 \lambda_{n}^{2} a_{n}^{2}\left(\sigma_{\varepsilon}^{2}+\left(\frac{\rho}{\tau_{\varepsilon}}\right)^{2} \sigma_{w}^{2}\right)\right)\right] .
\end{aligned}
$$

Proof: In the price schedule (2) replace $x_{n}$ by its expression given in (1) and after some rearranging, the price schedule can be written as

$$
p_{n}=\lambda_{n} x_{n}=\lambda_{n} a_{n} \tilde{v}+\lambda_{n} a_{n} \tilde{\varepsilon}-\lambda_{n} b_{n} \tilde{w} .
$$

Replace the above expression into the market maker's expected utility, we get

$$
\begin{aligned}
\Pi_{n}= & E\left[\left(\tilde{v}\left(\lambda_{n} a_{n}-1\right)+\lambda_{n} a_{n} \tilde{\varepsilon}-\lambda_{n} b_{n} \tilde{w}\right)\left(a_{n} \tilde{v}+a_{n} \tilde{\varepsilon}-b_{n} \tilde{w}\right)\right] \\
& -\frac{\rho_{m}}{2} \operatorname{var}\left[\left(\tilde{v}\left(\lambda_{n} a_{n}-1\right)+\lambda_{n} a_{n} \tilde{\varepsilon}-\lambda_{n} b_{n} \tilde{w}\right)\left(a_{n} \tilde{v}+a_{n} \tilde{\varepsilon}-b_{n} \tilde{w}\right)\right] .
\end{aligned}
$$

Developing and using the fact that all random variables are independent and have zero mean leads to

$$
\begin{aligned}
\Pi_{n}= & a_{n}\left(\lambda_{n} a_{n}-1\right) \sigma_{v}^{2}+\lambda_{n}\left(a_{n}^{2} \sigma_{\varepsilon}^{2}+b_{n}^{2} \sigma_{w}^{2}\right)-\frac{\rho_{m}}{2}\left[\left(a_{n}\left(\lambda_{n} a_{n}-1\right)\right)^{2} \operatorname{var}\left[\tilde{v}^{2}\right]\right. \\
& +\lambda_{n}^{2} b_{n}^{4} \operatorname{var}\left[(\tilde{w})^{2}\right]+\left(2 \lambda_{n} a_{n}-1\right)^{2}\left(a_{n}^{2} \operatorname{var}[\tilde{\operatorname{v}} \tilde{\varepsilon}]+b_{n}^{2} \operatorname{var}[\tilde{v} \tilde{w}]\right) \\
& \left.+4\left(\lambda_{n} a_{n} b_{n}\right)^{2} \operatorname{var}[\tilde{\varepsilon} \tilde{w}]+\lambda_{n}^{2} a_{n}^{4} \operatorname{var}\left[(\tilde{\varepsilon})^{2}\right]\right] .
\end{aligned}
$$

We need to compute all individual variances, using some basic statistics techniques, we have

$$
\begin{aligned}
& \operatorname{var}\left[\tilde{v}^{2}\right]=E\left[\tilde{v}^{4}\right]-E^{2}\left[\tilde{v}^{2}\right]=2 \sigma_{v}^{4}, \\
& \operatorname{var}[\tilde{v} \tilde{\varepsilon}]=\sigma_{v}^{2} \sigma_{\varepsilon}^{2}, \\
& \operatorname{var}[\tilde{v} \tilde{w}]=\sigma_{v}^{2} \sigma_{w}^{2}, \\
& \operatorname{var}[\tilde{\varepsilon} \tilde{w}]=\sigma_{\varepsilon}^{2} \sigma_{w}^{2}, \\
& \operatorname{var}\left[(\tilde{\varepsilon})^{2}\right]=2 \sigma_{\varepsilon}^{4} \text {, } \\
& \operatorname{var}\left[(\tilde{w})^{2}\right]=2 \sigma_{w}^{4} \text {. }
\end{aligned}
$$

Replace all the individual variances into the expression of the expected utility 
of profit (10), use the fact that $a_{n}=\frac{\tau_{\varepsilon}}{\rho} b_{n}$ after some simplifications the desired result is obtained.

Lemma 5. Given the linearity of both the market orders and the price schedule, given by (1) and (2) respectively, and given proposition 1, the expected utility of the trader is given by

$$
\begin{aligned}
\Pi= & E[W]-\frac{\rho}{2} \operatorname{var}[W] \\
= & \sum_{n=1}^{N} a_{n}\left[\sigma_{v}^{2}-\delta\left(\sigma_{v}^{2}+\sigma_{\varepsilon}^{2}+\frac{\rho^{2}}{\tau_{\varepsilon}^{2}} \sigma_{w}^{2}\right)\right] \\
& -\frac{\rho}{2}\left(\sum_{n=1}^{N} a_{n}\right)^{2}\left[2 \delta^{2}\left[\sigma_{v}^{4}+\sigma_{\varepsilon}^{4}+2 \sigma_{v}^{2} \sigma_{\varepsilon}^{2}+\frac{\rho^{2}}{\tau_{\varepsilon}^{2}} \sigma_{w}^{2}\left[2 \sigma_{v}^{2}+2 \sigma_{\varepsilon}^{2}+\frac{\rho^{2}}{\tau_{\varepsilon}^{2}} \sigma_{w}^{2}\right]\right]\right. \\
& \left.+\sigma_{v}^{2}\left((1-4 \delta)\left(\sigma_{v}^{2}+\sigma_{\varepsilon}^{2}+\frac{\rho^{2}}{\tau_{\varepsilon}^{2}} \sigma_{w}^{2}\right)+\sigma_{v}^{2}\right)\right] .
\end{aligned}
$$

Proof: The trader's expected utility is such that

$$
\Pi=E[W]-\frac{\rho}{2} \operatorname{var}[W]
$$

with

$$
W=\sum_{n=1}^{N} x_{n}\left(v-p_{n}\right)
$$

Using the expression of $p_{n}=\lambda_{n} x_{n}$ and the linearity of the market orders, the first term $E[W]$ is equal to

$$
E[W]=\sum_{n=1}^{N}\left[a_{n} \sigma_{v}^{2}\left(1-\lambda_{n} a_{n}\right)-\lambda_{n}\left(a_{n}^{2} \sigma_{\varepsilon}^{2}+b_{n}^{2} \sigma_{w}^{2}\right)\right] .
$$

We now turn to the computation of $\operatorname{var}[W]$. This variance can be rewritten as

$$
\operatorname{var}[W]=\operatorname{var}[C-A+B]
$$

with

$$
\begin{gathered}
A=\sum_{n=1}^{N} \lambda_{n} a_{n}\left(a_{n} s-b_{n} w\right) s, \\
B=\sum_{n=1}^{N} \lambda_{n} b_{n}\left(a_{n} s-b_{n} w\right) w, \\
C=\sum_{n=1}^{N}\left(a_{n} s-b_{n} w\right) v .
\end{gathered}
$$

The variance is then

$\operatorname{var}[W]=\operatorname{var}(A)+\operatorname{var}(B)+\operatorname{var}(C)-2 \operatorname{cov}(A, B)-2 \operatorname{cov}(A, C)+2 \operatorname{cov}(B, C)$.

The first term $\operatorname{var}(A)$ is equal to

$$
\operatorname{var}(A)=\operatorname{var}\left[\sum_{n=1}^{N} \lambda_{n} a_{n}^{2} s^{2}-\sum_{n=1}^{N} \lambda_{n} a_{n} b_{n} w s\right] .
$$


Simplifying and noting that

$$
\operatorname{cov}\left(\sum_{n=1}^{N} \lambda_{n} a_{n}^{2} s^{2}, \sum_{n=1}^{N} \lambda_{n} a_{n} b_{n} w s\right)=0,
$$

we get

$$
\operatorname{var}(A)=\operatorname{var}\left[\sum_{n=1}^{N} \lambda_{n} a_{n}^{2}\left(v^{2}+2 v \varepsilon+\varepsilon^{2}\right)\right]+\operatorname{var}\left[\sum_{n=1}^{N} \lambda_{n} a_{n} b_{n}(w v+w \varepsilon)\right] .
$$

Finally we obtain

$$
\operatorname{var}(A)=\left(\sum_{n=1}^{N} \lambda_{n} a_{n}^{2}\right)^{2}\left[2 \sigma_{v}^{4}+2 \sigma_{\varepsilon}^{4}+4 \sigma_{v}^{2} \sigma_{\varepsilon}^{2}\right]+\left(\sum_{n=1}^{N} \lambda_{n} a_{n} b_{n}\right)^{2} \sigma_{w}^{2}\left[\sigma_{v}^{2}+\sigma_{\varepsilon}^{2}\right] .
$$

The second term $\operatorname{var}(B)$ is equal to

$$
\operatorname{var}(B)=\operatorname{var}\left[\sum_{n=1}^{N} \lambda_{n} b_{n}\left(a_{n} s-b_{n} w\right) w\right] .
$$

Simplifying and as all random variables are independent and have zero expectation, we get

$$
\operatorname{var}(B)=\left(\sum_{n=1}^{N} \lambda_{n} a_{n} b_{n}\right)^{2} \sigma_{w}^{2}\left(\sigma_{v}^{2}+\sigma_{\varepsilon}^{2}\right)+\left(\sum_{n=1}^{N} \lambda_{n} b_{n}^{2}\right)^{2} 2 \sigma_{w}^{4} .
$$

The third term, $\operatorname{var}(C)$ is equal to

$$
\operatorname{var}(C)=\operatorname{var}\left(\sum_{n=1}^{N}\left(a_{n} s-b_{n} w\right) v\right) \text {. }
$$

This term can be simplified to

$$
\operatorname{var}(C)=\left(\sum_{n=1}^{N} a_{n}\right)^{2}\left(2 \sigma_{v}^{4}+\sigma_{\varepsilon}^{2} \sigma_{v}^{2}\right)+\left(\sum_{n=1}^{N} b_{n}\right)^{2} \sigma_{v}^{2} \sigma_{w}^{2} .
$$

We now compute all covariance terms.

The first covariance $\operatorname{cov}(A, B)$ is equal to

$$
\operatorname{cov}\left(\sum_{n=1}^{N} \lambda_{n} a_{n}^{2} s^{2}-\sum_{n=1}^{N} \lambda_{n} a_{n} b_{n} w s, \sum_{n=1}^{N} \lambda_{n} b_{n} a_{n} w s-\sum_{n=1}^{N} \lambda_{n} b_{n}^{2} w^{2}\right) .
$$

As all other covariance terms are equal to zero, we get that

$$
\begin{aligned}
& \operatorname{cov}(A, B)=-\left(\sum_{n=1}^{N} \lambda_{n} b_{n} a_{n}\right)^{2} \operatorname{cov}(w s, w s), \\
& \operatorname{cov}(A, B)=-\left(\sum_{n=1}^{N} \lambda_{n} b_{n} a_{n}\right)^{2} \sigma_{w}^{2}\left(\sigma_{v}^{2}+\sigma_{\varepsilon}^{2}\right) .
\end{aligned}
$$

The second term $\operatorname{cov}(A, C)$ is given by

$$
\operatorname{cov}\left(\sum_{n=1}^{N} \lambda_{n} a_{n}^{2} s^{2}-\sum_{n=1}^{N} \lambda_{n} a_{n} b_{n} w s, \sum_{n=1}^{N} a_{n}(v+\varepsilon) v-\sum_{n=1}^{N} b_{n} w v\right) .
$$

This simplifies to 


$$
\operatorname{cov}(A, C)=\left(\sum_{n=1}^{N} \lambda_{n} a_{n}^{2}\right)\left(\sum_{n=1}^{N} a_{n}\right)\left(2 \sigma_{v}^{4}+2 \sigma_{v}^{2} \sigma_{\varepsilon}^{2}\right)+\left(\sum_{n=1}^{N} \lambda_{n} a_{n} b_{n}\right)\left(\sum_{n=1}^{N} b_{n}\right) \sigma_{v}^{2} \sigma_{w}^{2} .
$$

The last term $\operatorname{cov}(B, C)$ is given by

$$
\operatorname{cov}\left(\sum_{n=1}^{N} \lambda_{n} b_{n} a_{n} s w-\sum_{n=1}^{N} \lambda_{n} b_{n}^{2} w^{2}, \sum_{n=1}^{N} a_{n}(v+\varepsilon) v-\sum_{n=1}^{N} b_{n} w v\right) .
$$

Ultimately, this leads to

$$
\operatorname{cov}(B, C)=-\left(\sum_{n=1}^{N} \lambda_{n} b_{n} a_{n}\right)\left(\sum_{n=1}^{N} b_{n}\right) \sigma_{v}^{2} \sigma_{w}^{2} .
$$

This leads to the expected profit equal to

$$
\begin{aligned}
\Pi= & E[W]-\frac{\rho}{2} \operatorname{var}[W] \\
= & \sum_{n=1}^{N}\left[a_{n} \sigma_{v}^{2}\left(1-\lambda_{n} a_{n}\right)-\lambda_{n}\left(a_{n}^{2} \sigma_{\varepsilon}^{2}+b_{n}^{2} \sigma_{w}^{2}\right)\right] \\
& -\frac{\rho}{2}\left[\left(\sum_{n=1}^{N} \lambda_{n} a_{n}^{2}\right)^{2}\left[2 \sigma_{v}^{4}+2 \sigma_{\varepsilon}^{4}+4 \sigma_{v}^{2} \sigma_{\varepsilon}^{2}\right]+\left(\sum_{n=1}^{N} \lambda_{n} a_{n} b_{n}\right)^{2} \sigma_{w}^{2}\left[\sigma_{v}^{2}+\sigma_{\varepsilon}^{2}\right]\right. \\
& +\left(\sum_{n=1}^{N} \lambda_{n} a_{n} b_{n}\right)^{2} \sigma_{w}^{2}\left(\sigma_{v}^{2}+\sigma_{\varepsilon}^{2}\right)+\left(\sum_{n=1}^{N} \lambda_{n} b_{n}^{2}\right)^{2} 2 \sigma_{w}^{4}+\left(\sum_{n=1}^{N} a_{n}\right)^{2}\left(2 \sigma_{v}^{4}+\sigma_{\varepsilon}^{2} \sigma_{v}^{2}\right) \\
& +\left(\sum_{n=1}^{N} b_{n}\right)^{2} \sigma_{v}^{2} \sigma_{w}^{2}+2\left(\sum_{n=1}^{N} \lambda_{n} b_{n} a_{n}\right)^{2} \sigma_{w}^{2}\left(\sigma_{v}^{2}+\sigma_{\varepsilon}^{2}\right) \\
& -2\left(\sum_{n=1}^{N} \lambda_{n} a_{n}^{2}\right)\left(\sum_{n=1}^{N} a_{n}\right)\left(2 \sigma_{v}^{4}+2 \sigma_{v}^{2} \sigma_{\varepsilon}^{2}\right)-2\left(\sum_{n=1}^{N} \lambda_{n} a_{n} b_{n}\right)\left(\sum_{n=1}^{N} b_{n}\right) \sigma_{v}^{2} \sigma_{w}^{2} \\
& \left.-2\left(\sum_{n=1}^{N} \lambda_{n} b_{n} a_{n}\right)\left(\sum_{n=1}^{N} b_{n}\right) \sigma_{v}^{2} \sigma_{w}^{2}\right] .
\end{aligned}
$$

This can be simplified to

$$
\begin{aligned}
& \Pi= E[W]-\frac{\rho}{2} \operatorname{var}[W] \\
&=\sum_{n=1}^{N}\left[a_{n} \sigma_{v}^{2}\left(1-\lambda_{n} a_{n}\right)-\lambda_{n}\left(a_{n}^{2} \sigma_{\varepsilon}^{2}+b_{n}^{2} \sigma_{w}^{2}\right)\right] \\
&-\frac{\rho}{2}\left[2\left(\sum_{n=1}^{N} \lambda_{n} a_{n}^{2}\right)^{2}\left[\sigma_{v}^{4}+\sigma_{\varepsilon}^{4}+2 \sigma_{v}^{2} \sigma_{\varepsilon}^{2}\right]+4\left(\sum_{n=1}^{N} \lambda_{n} a_{n} b_{n}\right)^{2} \sigma_{w}^{2}\left[\sigma_{v}^{2}+\sigma_{\varepsilon}^{2}\right]\right. \\
&+\left(\sum_{n=1}^{N} \lambda_{n} b_{n}^{2}\right)^{2} 2 \sigma_{w}^{4}+\left(\sum_{n=1}^{N} a_{n}\right)^{2}\left(2 \sigma_{v}^{4}+\sigma_{\varepsilon}^{2} \sigma_{v}^{2}\right)+\left(\sum_{n=1}^{N} b_{n}\right)^{2} \sigma_{v}^{2} \sigma_{w}^{2} \\
&\left.-4\left(\sum_{n=1}^{N} \lambda_{n} a_{n}^{2}\right)\left(\sum_{n=1}^{N} a_{n}\right)\left(\sigma_{v}^{4}+\sigma_{v}^{2} \sigma_{\varepsilon}^{2}\right)-4\left(\sum_{n=1}^{N} \lambda_{n} a_{n} b_{n}\right)\left(\sum_{n=1}^{N} b_{n}\right) \sigma_{v}^{2} \sigma_{w}^{2}\right] .
\end{aligned}
$$

Using the fact that $b_{n}=\frac{\rho}{\tau_{\varepsilon}} a_{n}$, the expected profit can be rewritten as 


$$
\begin{aligned}
\Pi= & E[W]-\frac{\rho}{2} \operatorname{var}[W] \\
= & \sum_{n=1}^{N}\left[a_{n} \sigma_{v}^{2}\left(1-\lambda_{n} a_{n}\right)-\lambda_{n}\left(a_{n}^{2} \sigma_{\varepsilon}^{2}+a_{n}^{2} \frac{\rho^{2}}{\tau_{\varepsilon}^{2}} \sigma_{w}^{2}\right)\right] \\
& -\frac{\rho}{2}\left[2\left(\sum_{n=1}^{N} \lambda_{n} a_{n}^{2}\right)^{2}\left[\sigma_{v}^{4}+\sigma_{\varepsilon}^{4}+2 \sigma_{v}^{2} \sigma_{\varepsilon}^{2}\right]+4\left(\sum_{n=1}^{N} \lambda_{n} a_{n} \frac{\rho}{\tau_{\varepsilon}} a_{n}\right)^{2} \sigma_{w}^{2}\left[\sigma_{v}^{2}+\sigma_{\varepsilon}^{2}\right]\right. \\
& +\left(\sum_{n=1}^{N} \lambda_{n} \frac{\rho^{2}}{\tau_{\varepsilon}^{2}} a_{n}^{2}\right)^{2} 2 \sigma_{w}^{4}+\left(\sum_{n=1}^{N} a_{n}\right)^{2}\left(2 \sigma_{v}^{4}+\sigma_{\varepsilon}^{2} \sigma_{v}^{2}\right)+\left(\sum_{n=1}^{N} \frac{\rho}{\tau_{\varepsilon}} a_{n}\right)^{2} \sigma_{v}^{2} \sigma_{w}^{2} \\
& \left.-4\left(\sum_{n=1}^{N} \lambda_{n} a_{n}^{2}\right)\left(\sum_{n=1}^{N} a_{n}\right)\left(\sigma_{v}^{4}+\sigma_{v}^{2} \sigma_{\varepsilon}^{2}\right)-4\left(\sum_{n=1}^{N} \lambda_{n} a_{n} \frac{\rho}{\tau_{\varepsilon}} a_{n}\right)\left(\sum_{n=1}^{N} \frac{\rho}{\tau_{\varepsilon}} a_{n}\right) \sigma_{v}^{2} \sigma_{w}^{2}\right] .
\end{aligned}
$$

Simplifying further

$$
\begin{aligned}
\Pi= & E[W]-\frac{\rho}{2} \operatorname{var}[W] \\
= & \sum_{n=1}^{N}\left[a_{n} \sigma_{v}^{2}\left(1-\lambda_{n} a_{n}\right)-\lambda_{n} a_{n}^{2}\left(\sigma_{\varepsilon}^{2}+\frac{\rho^{2}}{\tau_{\varepsilon}^{2}} \sigma_{w}^{2}\right)\right] \\
& -\frac{\rho}{2}\left[2\left(\sum_{n=1}^{N} \lambda_{n} a_{n}^{2}\right)^{2}\left[\sigma_{v}^{4}+\sigma_{\varepsilon}^{4}+2 \sigma_{v}^{2} \sigma_{\varepsilon}^{2}+2 \frac{\rho^{2}}{\tau_{\varepsilon}^{2}} \sigma_{w}^{2}\left[\sigma_{v}^{2}+\sigma_{\varepsilon}^{2}\right]+\frac{\rho^{4}}{\tau_{\varepsilon}^{4}} \sigma_{w}^{4}\right]\right. \\
& +\left(\sum_{n=1}^{N} a_{n}\right)^{2}\left(2 \sigma_{v}^{4}+\sigma_{\varepsilon}^{2} \sigma_{v}^{2}+\frac{\rho^{2}}{\tau_{\varepsilon}^{2}} \sigma_{v}^{2} \sigma_{w}^{2}\right) \\
& \left.-4\left(\sum_{n=1}^{N} \lambda_{n} a_{n}^{2}\right)\left(\sum_{n=1}^{N} a_{n}\right)\left(\sigma_{v}^{4}+\sigma_{v}^{2} \sigma_{\varepsilon}^{2}+\frac{\rho^{2}}{\tau_{\varepsilon}^{2}} \sigma_{v}^{2} \sigma_{w}^{2}\right)\right] .
\end{aligned}
$$

Given the fact that $\lambda_{n} a_{n}=\lambda_{j} a_{j} \forall j \neq n$. Let us assume that $\lambda_{n} a_{n}=\delta$, we can further simplify the expression to

$$
\begin{aligned}
\Pi= & E[W]-\frac{\rho}{2} \operatorname{var}[W] \\
= & \sum_{n=1}^{N}\left[a_{n} \sigma_{v}^{2}-\lambda_{n} a_{n}^{2}\left(\sigma_{v}^{2}+\sigma_{\varepsilon}^{2}+\frac{\rho^{2}}{\tau_{\varepsilon}^{2}} \sigma_{w}^{2}\right)\right] \\
& -\frac{\rho}{2}\left[2 \delta^{2}\left(\sum_{n=1}^{N} a_{n}\right)^{2}\left[\sigma_{v}^{4}+\sigma_{\varepsilon}^{4}+2 \sigma_{v}^{2} \sigma_{\varepsilon}^{2}+2 \frac{\rho^{2}}{\tau_{\varepsilon}^{2}} \sigma_{w}^{2}\left[\sigma_{v}^{2}+\sigma_{\varepsilon}^{2}\right]+\frac{\rho^{4}}{\tau_{\varepsilon}^{4}} \sigma_{w}^{4}\right]\right. \\
& \left.+\left(\sum_{n=1}^{N} a_{n}\right)^{2}\left(2 \sigma_{v}^{4}+\sigma_{\varepsilon}^{2} \sigma_{v}^{2}+\frac{\rho^{2}}{\tau_{\varepsilon}^{2}} \sigma_{v}^{2} \sigma_{w}^{2}\right)-4 \delta\left(\sum_{n=1}^{N} a_{n}\right)^{2}\left(\sigma_{v}^{4}+\sigma_{v}^{2} \sigma_{\varepsilon}^{2}+\frac{\rho^{2}}{\tau_{\varepsilon}^{2}} \sigma_{v}^{2} \sigma_{w}^{2}\right)\right] .
\end{aligned}
$$

This leads to 


$$
\begin{aligned}
\Pi= & E[W]-\frac{\rho}{2} \operatorname{var}[W] \\
= & \sum_{n=1}^{N} a_{n}\left[\sigma_{v}^{2}-\delta\left(\sigma_{v}^{2}+\sigma_{\varepsilon}^{2}+\frac{\rho^{2}}{\tau_{\varepsilon}^{2}} \sigma_{w}^{2}\right)\right] \\
& -\frac{\rho}{2}\left(\sum_{n=1}^{N} a_{n}\right)^{2}\left[2 \delta^{2}\left[\sigma_{v}^{4}+\sigma_{\varepsilon}^{4}+2 \sigma_{v}^{2} \sigma_{\varepsilon}^{2}+\frac{\rho^{2}}{\tau_{\varepsilon}^{2}} \sigma_{w}^{2}\left[2 \sigma_{v}^{2}+2 \sigma_{\varepsilon}^{2}+\frac{\rho^{2}}{\tau_{\varepsilon}^{2}} \sigma_{w}^{2}\right]\right]\right. \\
& \left.+\sigma_{v}^{2}\left((1-4 \delta)\left(\sigma_{v}^{2}+\sigma_{\varepsilon}^{2}+\frac{\rho^{2}}{\tau_{\varepsilon}^{2}} \sigma_{w}^{2}\right)+\sigma_{v}^{2}\right)\right] .
\end{aligned}
$$

\section{Proof of Proposition 1}

Proof. Given her order flow and given the trader's market order, each market marker $n$ maximizes her expected utility with respect to $\lambda_{n}$ such that

$$
\begin{aligned}
& \max _{\lambda_{n}} a_{n} \sigma_{v}^{2}\left(\lambda_{n} a_{n}-1\right)+\lambda_{n} a_{n}^{2}\left(\sigma_{\varepsilon}^{2}+\left(\frac{\rho}{\tau_{\varepsilon}}\right)^{2} \sigma_{w}^{2}\right)-\frac{\rho_{m}}{2} a_{n}^{2}\left[2 \sigma_{v}^{4}\left(\lambda_{n} a_{n}-1\right)^{2}\right. \\
&\left.+\left(\sigma_{\varepsilon}^{2}+\left(\frac{\rho}{\tau_{\varepsilon}}\right)^{2} \sigma_{w}^{2}\right)\left(\sigma_{v}^{2}\left(2 \lambda_{n} a_{n}-1\right)^{2}+2 \lambda_{n}^{2} a_{n}^{2}\left(\sigma_{\varepsilon}^{2}+\left(\frac{\rho}{\tau_{\varepsilon}}\right)^{2} \sigma_{w}^{2}\right)\right)\right]
\end{aligned}
$$

given that

$$
a_{n}=\frac{\tau_{\varepsilon} \prod_{\substack{i=1 \\ i \neq n}}^{N} \lambda_{i}}{\rho \sum_{\substack{j=1 \\ i=1 \\ i \neq j}}^{N} \lambda_{i}+2\left(\tau_{\varepsilon}+\tau_{v}\right) \prod_{i=1}^{N} \lambda_{i}},
$$

with $a_{n}=\frac{\tau_{\varepsilon}}{\rho} b_{n}$.

Provided that $\lambda_{n} \neq 0$, the FOC of the above program can be simplified after some tedious computations to

$$
\alpha_{4} \lambda_{n}^{4}+\alpha_{3} \lambda_{n}^{3}+\alpha_{2} \lambda_{n}^{2}+\alpha_{1} \lambda_{n}+\alpha_{0}=0
$$

with

$$
\begin{gathered}
\alpha_{4}=\frac{8\left(\tau_{v}+\tau_{\varepsilon}\right)^{3}}{\tau_{v} \tau_{w}}\left(-\rho^{2} \tau_{w}^{-1}+\tau_{\varepsilon}\left(1+\tau_{\varepsilon} \tau_{v}^{-1}\right)\right), \\
\alpha_{0}=\frac{N^{2}(N-1) \rho^{3}}{\tau_{v}^{2} \tau_{w}}\left(\rho^{2} \rho_{m} \tau_{v}+N \rho \tau_{v} \tau_{w} \tau_{\varepsilon}+\rho_{m} \tau_{w} \tau_{\varepsilon}\left(\tau_{v}+2 \tau_{\varepsilon}\right)\right), \\
\frac{\alpha_{3} \tau_{v}^{2} \tau_{w}^{2}}{4\left(\tau_{v}+\tau_{\varepsilon}\right)}=\rho_{m} \tau_{v}^{2} \rho^{4}+(2-3 N) \tau_{v}^{2} \tau_{w}\left(\tau_{v}+\tau_{\varepsilon}\right) \rho^{3}+2\left(\tau_{v}+\tau_{\varepsilon}\right) \tau_{v}^{2} \tau_{w} \rho_{m} \rho^{2} \\
+5\left(\tau_{v}+\tau_{\varepsilon}\right)^{2} \tau_{v} \tau_{\varepsilon} \tau_{w}^{2} N \rho+\left(\tau_{v}+\tau_{\varepsilon}\right)^{2}\left(2 \tau_{v}+\tau_{\varepsilon}\right) \tau_{w}^{2} \tau_{\varepsilon} \rho_{m},
\end{gathered}
$$




$$
\begin{aligned}
& \frac{\alpha_{2} \tau_{v}^{2} \tau_{w}^{2}}{2 \rho}=(-2+N) \rho_{m} \tau_{v}^{2} \rho^{4}+\left(\tau_{v}+\tau_{\varepsilon}\right) \tau_{v}^{2} \tau_{w} N(4-3 N) \rho^{3} \\
&+2\left(\tau_{v}+\tau_{\varepsilon}\right) \tau_{w} \tau_{v}^{2} \rho_{m}(-1+3 N) \rho^{2} \\
&+\left(\tau_{v}+\tau_{\varepsilon}\right)^{2} \tau_{v} \tau_{\varepsilon} \tau_{w}^{2} N(-2+9 N) \rho \\
&+\left(\tau_{v}+\tau_{\varepsilon}\right)^{2} \tau_{w}^{2} \tau_{\varepsilon} \rho_{m}\left((-2+6 N) \tau_{v}+5 N \tau_{\varepsilon}\right) \\
& \frac{\alpha_{1} \tau_{v}^{2} \tau_{w}}{N \rho^{2}=} \tau_{v}^{2} N(2-N) \rho^{3}+2 \tau_{v} \rho_{m}\left(\tau_{v}(3-2 N)+\tau_{\varepsilon}(N+1)\right) \rho^{2} \\
&+N \tau_{v} \tau_{w} \tau_{\varepsilon}\left(\tau_{v}+\tau_{\varepsilon}\right)(-4+7 N) \rho \\
&+2 \rho_{m} \tau_{w} \tau_{\varepsilon}\left(-\left(2 \tau_{v}^{2}+3 \tau_{v} \tau_{\varepsilon}+\tau_{\varepsilon}^{2}\right)+N\left(3 \tau_{v}^{2}+7 \tau_{v} \tau_{\varepsilon}+4 \tau_{\varepsilon}^{2}\right)\right)
\end{aligned}
$$

The SOC is strictly negative and therefore the solution, if any, to the above FOC is a maximum.

We now analyze the above expression (11). In order to determine whether it has a solution and as it is a polynomial of degree 4 , we analyze the sign of the coefficient of that equation.

First of all it can be seen that the coefficient $\alpha_{0}$ is always positive if $N>1$. It can also be shown that if $\rho^{2} \tau_{w}^{-1} \leq \tau_{\varepsilon}\left(1+\tau_{\varepsilon} \tau_{v}^{-1}\right)$, the coefficient $\alpha_{4}$ is non-negative. Using some basic algebra, it can be proved that it implies that all other coefficients of the above polynomial are non-negative. In that case the FOC condition has no solution.

When $\rho^{2} \tau_{w}^{-1}>\tau_{\varepsilon}\left(1+\tau_{\varepsilon} \tau_{v}^{-1}\right)$ and $N>1$, the coefficient $\alpha_{4}$ is strictly negative and as the coefficient $\alpha_{0}$ is positive this guarantees the existence of a positive root for the FOC. When $N=1, \alpha_{4}$ is still strictly negative, whereas as $\alpha_{0}=0$ and $\alpha_{1}>0$, this also leads to the existence of a solution.

In order to determine the unicity of the root, we have to analyze the sign of the remaining coefficients: $\alpha_{3}, \alpha_{2}, \alpha_{1}$. We start with the coefficient $\alpha_{3}$.

- Coefficient $\alpha_{3}$.

The parameter $\alpha_{3}$ can be written as follows

$$
\frac{\alpha_{3} \tau_{v}^{2} \tau_{w}^{2}}{4\left(\tau_{v}+\tau_{\epsilon}\right)}=c_{4} \rho^{4}+c_{3} \rho^{3}+c_{2} \rho^{2}+c_{1} \rho+c_{0},
$$

with

$$
\begin{gathered}
c_{4}=\rho_{m} \tau_{v}^{2}, \quad c_{3}=(2-3 N) \tau_{v}^{2} \tau_{w}\left(\tau_{v}+\tau_{\epsilon}\right), \quad c_{2}=2\left(\tau_{v}+\tau_{\epsilon}\right) \tau_{v}^{2} \tau_{w} \rho_{m}, \\
c_{1}=5\left(\tau_{v}+\tau_{\epsilon}\right)^{2} \tau_{v} \tau_{\epsilon} \tau_{w}^{2} N \text { and } c_{0}=\left(\tau_{v}+\tau_{\epsilon}\right)^{2}\left(2 \tau_{v}+\tau_{\epsilon}\right) \tau_{w}^{2} \tau_{\epsilon} \rho_{m} .
\end{gathered}
$$

As we want to find the sign of the RHS, we consider it as a function of $\rho$ and we denote it $f_{3}(\rho)$.

The sign of these coefficients can easily be determined: $c_{4}>0, c_{2}>0$, $c_{1}>0, c_{0}>0, c_{3}<0$.

The first and second derivatives of that function with respect $\rho$ are given by

$$
\begin{gathered}
f_{3}^{\prime}(\rho)=4 c_{4} \rho^{3}+3 c_{3} \rho^{2}+2 c_{2} \rho+c_{1}, \\
f_{3}^{\prime \prime}(\rho)=12 c_{4} \rho^{2}+6 c_{3} \rho+2 c_{2} .
\end{gathered}
$$


We are looking for the sign of $f_{3}^{\prime \prime}$ and compute the discriminant of $f_{3}^{\prime \prime}$

$$
\Delta_{3}=12\left(3 c_{3}^{2}-8 c_{4} c_{2}\right) \text {. }
$$

Plugging in the expressions of $c_{4}, c_{3}$ and $c_{2}$ and after some computations, the discriminant is given by

$$
\Delta_{3}=12 \tau_{v}^{4} \tau_{w}\left(\tau_{v}+\tau_{\epsilon}\right)\left(3 \tau_{w}\left(\tau_{v}+\tau_{\epsilon}\right)(-2+3 N)^{2}-16 \rho_{m}^{2}\right) .
$$

The sign of $\Delta_{3}$ changes with $\rho_{m}$. We first determine the value of $\rho_{m}$ for which the discriminant is equal to zero

$$
\rho_{m_{3}}=\frac{-2+3 N}{4} \sqrt{3 \tau_{w}\left(\tau_{v}+\tau_{\epsilon}\right)}
$$

when $\rho_{m}>\rho_{m_{3}}$, the discriminant is always negative and $f_{3}^{\prime \prime}$ is always of the sign of $c_{4}>0$. This implies that $f_{3}^{\prime}$ is always increasing and moreover $f_{3}^{\prime}(0)=c_{1}>0$. As a result $f_{3}^{\prime}$ is strictly positive implying that $f_{3}(\rho)$ is an increasing function. As $f_{3}(0)=c_{0}>0$, the coefficient $\alpha_{3}$ is always positive.

When $\rho_{m}<\rho_{m_{3}}$, the discriminant is positive and $f_{3}^{\prime \prime}$ admits two positive roots given by

$$
\begin{aligned}
& \rho_{3}^{*}=\frac{3 \tau_{w}\left(\tau_{v}+\tau_{\epsilon}\right)(-2+3 N)-\sqrt{3 \tau_{w}\left(\tau_{v}+\tau_{\epsilon}\right)\left(3 \tau_{w}\left(\tau_{v}+\tau_{\epsilon}\right)(-2+3 N)^{2}-16 \rho_{m}^{2}\right)}}{12 \rho_{m}}, \\
& \rho_{3}^{* *}=\frac{3 \tau_{w}\left(\tau_{v}+\tau_{\epsilon}\right)(-2+3 N)+\sqrt{3 \tau_{w}\left(\tau_{v}+\tau_{\epsilon}\right)\left(3 \tau_{w}\left(\tau_{v}+\tau_{\epsilon}\right)(-2+3 N)^{2}-16 \rho_{m}^{2}\right)}}{12 \rho_{m}} .
\end{aligned}
$$

The following table can be given in order to find the sign of $a_{3}$

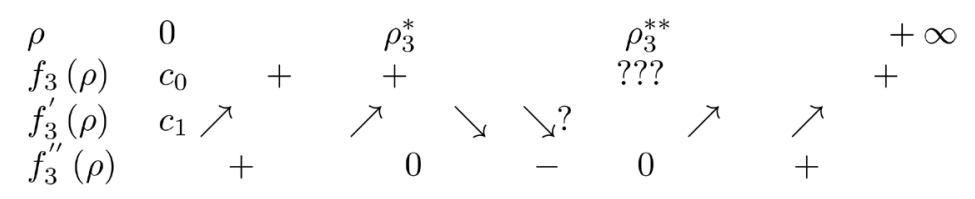

That table implies that for $\rho \leq \rho_{3}^{*}$ the coefficient $\alpha_{3}$ is strictly positive as well as for $\rho$ large. For intermediate $\rho$ and by continuity we can say that when $\rho$ is close to $\rho_{3}^{*}$ but larger we still have that $\alpha_{3}$ is positive. However as $\rho$ gets closer to $\rho_{3}^{* *}, f_{3}^{\prime}(\rho)$ decreases and can potentially become negative as it becomes negative that means that $f_{3}(\rho)$ would decrease with $\rho$ and could also become negative. For $\rho>\rho_{3}^{*}$, the function $f_{3}(\rho)$ admits a minimum at $\rho_{3}^{* *}$. This leaves the possibility to obtain two roots, if the function admits a negative value at $\rho_{3}^{* *}$.

\section{- Coefficient $\alpha_{2}$.}

We proceed as for $\alpha_{3}$.

Provided $\rho \neq 0$, the sign of $\alpha_{2}$ is equivalent to the sign of the expression in between brackets. Let us define that expression as being a function of $\rho$ and let us denote it $f_{2}(\rho)$. This function is given by

$$
f_{2}(\rho)=d_{4} \rho^{4}+d_{3} \rho^{3}+d_{2} \rho^{2}+d_{1} \rho+d_{0},
$$

with 


$$
\begin{gathered}
d_{4}=(-2+N) \rho_{m} \tau_{v}^{2}, \\
d_{3}=\left(\tau_{v}+\tau_{\epsilon}\right) \tau_{v}^{2} \tau_{w} n(4-3 N), \\
d_{2}=2\left(\tau_{v}+\tau_{\epsilon}\right) \tau_{w} \tau_{v}^{2} \rho_{m}(-1+3 N), \\
d_{1}=\left(\tau_{v}+\tau_{\epsilon}\right)^{2} \tau_{v} \tau_{\epsilon} \tau_{w}^{2} N(-2+9 N)
\end{gathered}
$$

and finally

$$
d_{0}=\left(\tau_{v}+\tau_{\epsilon}\right)^{2} \tau_{w}{ }^{2} \tau_{\epsilon} \rho_{m}\left((-2+6 N) \tau_{v}+5 N \tau_{\epsilon}\right) .
$$

First of all the sign of $d_{2}, d_{1}$, and $d_{0}$ can be established as being positive for any values of $n$.

It can be seen that for $N \leq 2, d_{3}$ can change sign. However, we can establish that there is only one change in the sign of the coefficients for the polynomial $f_{2}(\rho)$. This implies one positive root for the polynomial (we call it $\hat{\rho}_{2}$ ). For $\rho \geq \hat{\rho}_{2} \quad\left(\rho<\hat{\rho}_{2}\right)$, we obtain that $\alpha_{2} \leq 0 \quad\left(\alpha_{2}>0\right)$.

When $N \geq 3$, the analysis is more complex. Let us compute the first and second derivative of that function with respect to $\rho$

$$
\begin{gathered}
f_{2}^{\prime}(\rho)=4 d_{4} \rho^{3}+3 d_{3} \rho^{2}+2 d_{2} \rho+d_{1} \\
f_{2}^{\prime \prime}(\rho)=12 d_{4} \rho^{2}+6 d_{3} \rho+2 d_{2},
\end{gathered}
$$

As we are looking for the sign of $f^{\prime \prime}(\rho)$ we compute its discriminant

$$
\Delta_{2}=12\left(3 d_{3}^{2}-8 d_{4} d_{2}\right) \text {. }
$$

Plugging in the values of $c_{4}, c_{3}$ and $c_{2}$ and after some simplifications the following expression is obtained

$$
\Delta_{2}=12\left(\tau_{v}+\tau_{\epsilon}\right) \tau_{v}^{4} \tau_{w}\left[3\left(\tau_{v}+\tau_{\epsilon}\right) \tau_{w} N^{2}(4-3 N)^{2}-16(-2+N)(-1+3 N) \rho_{m}^{2}\right] .
$$

Given that expression we can define $\rho_{m_{2}}$ as the value of $\rho_{m}$ such that the discriminant is equal to 0 :

$$
\rho_{m_{2}}=\frac{(3 N-4) N \sqrt{3 \tau_{w}\left(\tau_{v}+\tau_{\epsilon}\right)}}{4 \sqrt{(-2+N)(-1+3 N)}} .
$$

For any values of $\rho_{m}>\rho_{m_{2}}$ the discriminant is negative and $f_{2}^{\prime \prime}$ is always positive. In that case $f_{2}^{\prime}$ is always increasing with $f^{\prime}(0)=d_{1}>0$. This implies that $f^{\prime}(\rho)$ is always positive which in turns imply that, as $f(0)=d_{0}>0$, the coefficient $\alpha_{2}$ is always positive.

When $\rho_{m}<\rho_{m_{2}} \quad \Delta_{2}$ is positive and $f_{2}^{\prime \prime}$ admits two positive roots

$\rho_{2}^{*}=$

$$
\begin{aligned}
& \frac{3\left(\tau_{v}+\tau_{\epsilon}\right) \tau_{w} N(3 N-4)-\sqrt{3\left(\tau_{v}+\tau_{\epsilon}\right)\left[3\left(\tau_{v}+\tau_{\epsilon}\right) \tau_{w} N^{2}(4-3 N)^{2}-16(-2+N)(-1+3 N) \rho_{m}^{2}\right]}}{12(-2+N) \rho_{m}}, \\
& \rho_{2}^{* *}= \\
& \frac{3\left(\tau_{v}+\tau_{\epsilon}\right) \tau_{w} N(3 N-4)+\sqrt{3\left(\tau_{v}+\tau_{\epsilon}\right)\left[3\left(\tau_{v}+\tau_{\epsilon}\right) \tau_{w} N^{2}(4-3 N)^{2}-16(-2+N)(-1+3 N) \rho_{m}^{2}\right]}}{12(-2+N) \rho_{m}} .
\end{aligned}
$$


The following table can be used in order to determine the sign of $\alpha_{2}$

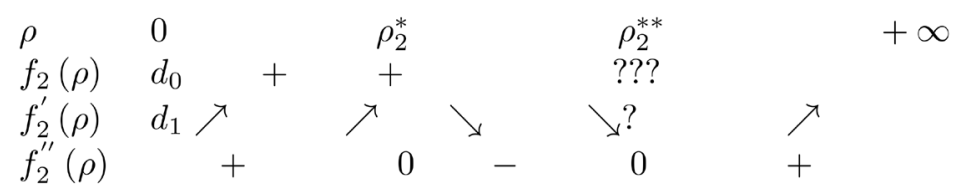

That table implies that for $\rho \leq \rho_{2}^{*}$ the coefficient $\alpha_{2}$ is strictly positive as well as for $\rho$ large. For intermediate $\rho$ and by continuity we can say that when $\rho$ is close to $\rho_{2}^{*}$ but larger we still have that $\alpha_{2}$ is positive. However as $\rho$ gets closer to $\rho_{2}^{* *}, f_{2}^{\prime}(\rho)$ decreases and could potentially become negative as it becomes negative that means that $f_{2}(\rho)$ would decrease with $\rho$ and could also become negative. For $\rho>\rho_{2}^{*}$, the function $f_{2}(\rho)$ admits a minimum at $\rho_{2}^{* *}$. This leaves the possibility to obtain two roots, if the function admits a negative value at $\rho_{2}^{* *}$.

- Coefficient $\alpha_{1}$.

We rewrite $\alpha_{1}$ as

$$
\frac{\alpha_{1} \tau_{v}^{2} \tau_{w}^{2}}{\rho^{2}}=\tau_{v}^{2} N(2-N) \rho^{3}+e_{2} \rho^{2}+e_{1} \rho+e_{0}
$$

with

$$
\begin{gathered}
e_{2}=2 \tau_{v} \rho_{m}\left(\tau_{v}(3-2 N)+\tau_{\epsilon}(N+1)\right), \\
e_{1}=N \tau_{v} \tau_{w} \tau_{\epsilon}\left(\tau_{v}+\tau_{\epsilon}\right)(-4+7 N)
\end{gathered}
$$

and finally

$$
e_{0}=2 \rho_{m} \tau_{w} \tau_{\varepsilon}\left(-\left(2 \tau_{v}^{2}+3 \tau_{v} \tau_{\epsilon}+\tau_{\epsilon}^{2}\right)+N\left(3 \tau_{v}^{2}+7 \tau_{v} \tau_{\epsilon}+4 \tau_{\epsilon}^{2}\right)\right)
$$

It can be seen that $e_{1}$ and $e_{0}$ are always positive independently of the value of $N$.

For $N=1$, the coefficient in front of $\rho^{3}$ is strictly positive as well as $e_{2}$. Leading to the fact $\alpha_{1}$ is strictly positive in that case.

For $N>1$, the sign of $\alpha_{1}$ is not straightforward.

When $N=2$, the expression above is a second degree polynomial where $e_{2}$ can either be positive or negative. When $-\tau_{v}+3 \tau_{\varepsilon}>0, \alpha_{1}$ is still positive. When $-\tau_{v}+3 \tau_{\varepsilon}<0$, the polynomial admits two roots (one positive and one negative leading to the fact that $\alpha_{1}$ is non-negative for values of $\left.\left.\rho \in\right] 0, \rho_{1}^{* *}\right]$ and positive for $\rho>\rho_{1}^{* *}$ if $\rho_{1}^{* *}$ is that positive root.

When $N \geq 3$, we consider as before the RHS as a function of $\rho$ and we denote it $f_{1}(\rho)$. The only coefficient that might change its sign is $e_{2}$. When $\tau_{v}(3-2 N)+\tau_{\epsilon}(N+1)<0$ it is negative and positive otherwise. However it can be seen from the expression of $\alpha_{1}$ that we have only one change in sign for the coefficients of the polynomial. This implies that $\alpha_{1}$ admits only one positive root, called $\hat{\rho}_{1}$. When $\rho \geq \hat{\rho}_{1}$, we have that $\alpha_{1} \leq 0$ and when $\rho<\hat{\rho}_{1}$, we obtain that $\alpha_{1}>0$.

- Comparison of thresholds for $N \geq 3$

$$
\rho_{m_{3}}=\sqrt{3 \tau_{w}\left(\tau_{v}+\tau_{\epsilon}\right)} \frac{-2+3 N}{4}
$$




$$
\rho_{m_{2}}=\sqrt{\frac{3 \tau_{w}\left(\tau_{v}+\tau_{\epsilon}\right)}{(-2+N)(-1+3 N)}} \frac{N(3 N-4)}{4}
$$

After some algebra and setting $\tau_{w}=\tau_{v}=\tau_{\epsilon}=1$, it can be proved that finding the sign $18 N^{4}-75 N^{\beta}+98 N^{2}-52 N+8$ is equivalent to find how $\rho_{m_{3}}$ compares to $\rho_{m_{2}}$. When $18 N^{4}-75 N^{\beta}+98 N^{2}-52 N+8>0$, it is equivalent to $\rho_{m_{2}}<\rho_{m_{3}}$.

The above inequality is correct for $N \geq 3$ and therefore leads to the fact that for $N \geq 3$ we have $\rho_{m_{3}}>\rho_{m_{2}}$.

- Comparison of $\alpha_{3}$ and $\alpha_{2}$

It can be shown that when $N \geq 3 \quad \alpha_{2}>\alpha_{3}$. This leads to the fact that when both expressions, $\alpha_{3}$ and $\alpha_{2}$, admit two positive roots, the positive roots for $\alpha_{2}$ are in between the two positive roots of $\alpha_{3}$. When $N<2$, such a comparison cannot be established.

\section{- Summary of our proof}

It can be established that for some parameters value the FOC admits only one solution. This happens when there is only change of sign for the parameters ( $\alpha$ s) of the polynomial. This establishes the unicity of the solution. For all other cases, where the unicity cannot be formally established, this happens when we have more than one change in sign for the $\alpha$ s of the expression (11). We use numerical simulations and always find that it is the case that the solution is unique.

The following three graphs (displayed as Figure 13) show possible configurations for different number of market makers. Each graph presents the cases where the unicity can be formally established (Unicity established) and where it cannot be formally established (Unicity not established) as a function of $\rho$ and $\rho_{m}$. The three graphs are not giving an exhaustive list of the different cases. For a given number of market makers, $N$, we have several cases describing all cases would be very long and not interesting per se, we have then chosen not to show them here. However an exhaustive list of all possible configurations is available upon request from the authors.

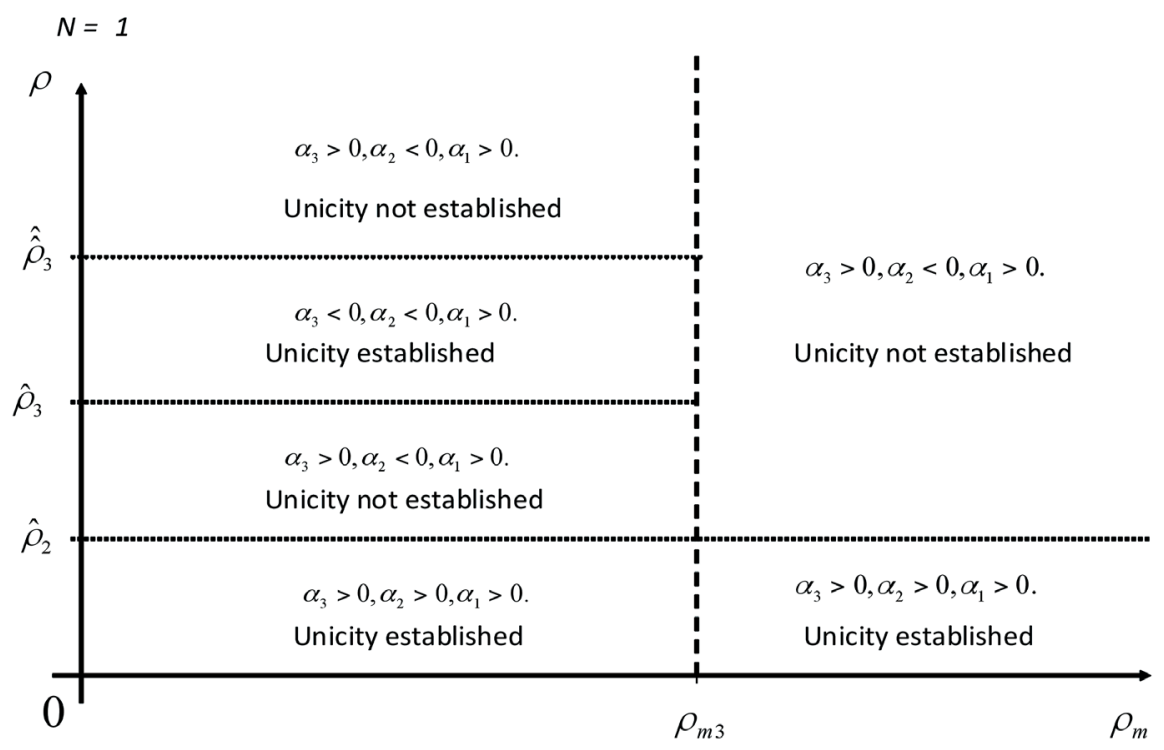



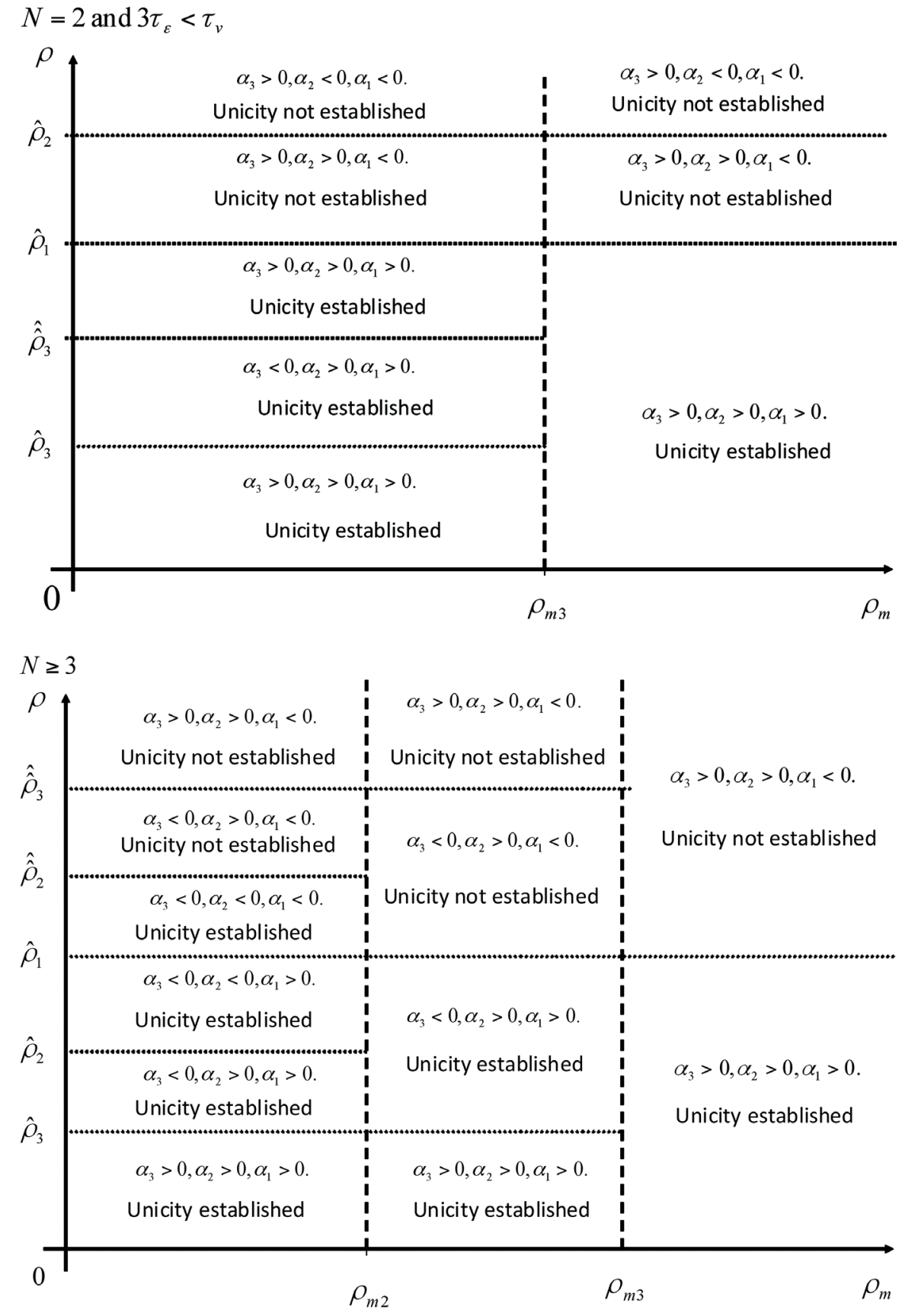

Figure 13. The figures show the cases for which the unicity can be established formally and cases for which it can not be as a function of $\rho_{m}$ and $\rho$. The figures correspond to the case where $N=1, N=2$ and $N=3$. 
Submit or recommend next manuscript to SCIRP and we will provide best service for you:

Accepting pre-submission inquiries through Email, Facebook, LinkedIn, Twitter, etc. A wide selection of journals (inclusive of 9 subjects, more than 200 journals)

Providing 24-hour high-quality service

User-friendly online submission system

Fair and swift peer-review system

Efficient typesetting and proofreading procedure

Display of the result of downloads and visits, as well as the number of cited articles Maximum dissemination of your research work

Submit your manuscript at: http://papersubmission.scirp.org/

Or contact jmf@scirp.org 